\title{
Stabilization and Synchronization of Uncertain Zhang System by Means of Robust Adaptive Control
}

\author{
J. Humberto Pérez-Cruz (10) \\ SEPI-ESIME-Azcapotzalco, Instituto Politécnico Nacional, Ciudad de México 02250, Mexico \\ Correspondence should be addressed to J. Humberto Pérez-Cruz; jhperez@ipn.mx
}

Received 19 August 2018; Revised 22 October 2018; Accepted 1 November 2018; Published 2 December 2018

Academic Editor: Ludovico Minati

Copyright (C) 2018 J. Humberto Pérez-Cruz. This is an open access article distributed under the Creative Commons Attribution License, which permits unrestricted use, distribution, and reproduction in any medium, provided the original work is properly cited.

Standard adaptive control is the preferred approach for stabilization and synchronization of chaotic systems when the structure of such systems is a priori known but the parameters are unknown. However, in the presence of unmodeled dynamics and/or disturbance, this approach is not effective anymore due to the drift of the parameter estimations, which eventually causes the instability of the closed-loop system. In this paper, a robustifying term, which consists of a saturation function, is used to avoid this problem. The robustifying term is added to the adaptive control law. Consequently, the learning law is also modified. The boundedness of the states and the parameter estimations is rigorously and thoroughly proven by means of Lyapunov like analysis based on Barbalat's lemma. On these new conditions, the convergence to zero cannot be achieved due to the presence of unmodeled dynamics and/or disturbance. However, it is still possible to guarantee the asymptotic convergence to a bounded zone around zero. The width of this zone can be adjusted by the designer. The performance of this robust approach is verified by numerical simulations. Although, for simplicity, this strategy is only applied to the stabilization and synchronization of Zhang system, the procedure can easily be generalized to a broad class of chaotic systems.

\section{Introduction}

Chaotic systems present interesting and peculiar features such as very high sensitivity to initial conditions, boundedness of solutions, and a rich dynamic behavior [1-6]. In particular, due to the first property, the long-term prediction of the dynamic behavior of these systems is not possible. However, it is still possible to modify the behavior of such systems by means of a proper control input. This fact is the basis of successful applications in fields as optics [7-10], secure communications [11-23], finance [5,24-28], power systems [29-33], electrical machinery [34-36], and so on.

Basically, two cases can be distinguished for control of chaotic systems: (a) chaos suppression and (b) synchronization $[37,38]$. In the first case, the states of a chaotic system tend to an equilibrium point, generally the origin, by means of a proper control law. In the second case, a chaotic system with control inputs known as slave must follow the dynamics of an autonomous chaotic system known as master. Both systems should produce the same response in spite of the different initial conditions [39]. This problem can be posed as the stabilization of the difference between the states of slave system and master system (synchronization error). From this point of view, synchronization can be simply considered as a generalization of stabilization problem [40].

In technical literature, various strategies have been proposed for control of chaotic systems [41-48]. Three approaches have commonly been used when both the structure and the parameters of the system are known: linear feedback control [49-53], nonlinear control [54-58], and active control [59-68]. In the first approach, the control input is formed by proportional feedback either of the system state or of the synchronization error according to the objective: stabilization or synchronization, respectively. In nonlinear control, the control input is designed in such a way that, given a Lyapunov function candidate $V$, the first time derivative $\dot{V}$ is negative definite. In this approach, the control input may differ depending on the particular selection of the designer. The active control consists of the compensation of nonlinearities and the decoupling of the equations that describe 
the dynamics of a system or of the synchronization error to achieve its stabilization [69]. The use of this technique always produces the same results independently of the designer. This technique can be considered as a special kind of feedback linearization [70].

On the other hand, when the structure is known but the parameters of the systems are unknown, the preferred approach is adaptive control [71-75]. This method can be considered as a generalization of active control. As in this case, the parameters are unknown, the compensation of nonlinearities and the decoupling of the equations must be carried out by using the estimations of such parameters. Consequently, an updating law should also be designed. A Lyapunov-like approach based on Barbalat's lemma is the main tool to show the closed-loop stability of this kind of systems. By using this method, the Lorenz and Chen systems and modified Chua and Rössler systems could be synchronized successfully in [76]. In [77], the stabilization with respect to zero equilibrium and other non-zero equilibria and synchronization of Liu system were accomplished by means of the adaptive approach. In [78], Chen and Genesio systems were synchronized using active and adaptive control for the case with known and unknown parameters, respectively. The synchronization of an uncertain hyperchaotic Lorenz system and an uncertain hyperchaotic Lü system was investigated in [79]. In [80], the adaptive control and synchronization of Rössler prototype-4 system were considered. In [71, 73], the adaptive control was applied to the synchronization of an uncertain chaotic Lorenz-Stenflo system and of uncertain TSUCS and Lü unified chaotic systems, respectively.

All the aforementioned works considered only the case when the parameters are unknown. However, in practical situations, unmodeled dynamics and/or disturbance could be additionally present. Under this more realistic condition, the control laws designed in [66-75] are not effective anymore since the response is deteriorated, the estimations of the parameters start to grow unboundedly, and eventually the closed-loop system becomes unstable. To overcome this problem, in this paper, a robustifying term, which consists of a saturation function, is used in the control law. Consequently, the corresponding learning law for parameter estimations is also modified. For simplicity, the attention is focused on a new chaotic system proposed in [81]. However, the explained strategy can be easily applied to a broad class of chaotic systems.

\section{Zhang System and Problem Description}

Zhang presented in [81] a new third order chaotic system formed by two linear terms, three cross-product terms, and a unique cubic term. This system is described by

$$
\begin{aligned}
& \dot{x}=-a x+b y z \\
& \dot{y}=-c y^{3}+d x z \\
& \dot{z}=f z-g x y
\end{aligned}
$$

where $x, y$, and $z$ are the system states and $a, b, c, d, f$, and $g$ are constant parameters. This system shows a chaotic behavior for the values $a=2, b=10, c=6.1, d=3, f=3$, and $g=1$ and the initial condition $x(0)=0.5, y(0)=0$, and $z(0)=0.5$. This phenomenon can be appreciated qualitatively in Figure 1 where the phase planes and the phase space are presented for four-scroll chaotic attractor of system (1). A more detailed description of the properties of system (1) can be founded in [81].

Certainly, if no external influence is applied to system (1), then its chaotic behavior cannot be suppressed. Thus, for trying to achieve this objective, it is necessary first to modify system (1) in the following way:

$$
\begin{aligned}
& \dot{x}=-a x+b y z+\mu_{1} \\
& \dot{y}=-c y^{3}+d x z+\mu_{2} \\
& \dot{z}=f z-g x y+\mu_{3}
\end{aligned}
$$

where $\mu_{1}, \mu_{2}$, and $\mu_{3}$ are control inputs. The stabilization problem for system (2) consists of finding a proper control law such that the states of this system tend to zero independently of the initial condition. Besides, the parameters of system (2) will be considered unknown throughout this paper.

Another problem (which could be considered as a generalization of stabilization) is the synchronization of chaotic systems. In its simpler conception, that is, master-slave configuration, a slave chaotic system with control inputs must follow the dynamic behavior of an autonomous master chaotic system. For system (1), the corresponding master system can be represented simply as

$$
\begin{aligned}
& \dot{x}_{m}=-a x_{m}+b y_{m} z_{m} \\
& \dot{y}_{m}=-c y_{m}^{3}+d x_{m} z_{m} \\
& \dot{z}_{m}=f z_{m}-g x_{m} y_{m}
\end{aligned}
$$

where the subscript $m$ denotes "master". The corresponding slave system for system (1) is given by

$$
\begin{aligned}
& \dot{x}_{s}=-a x_{s}+b y_{s} z_{s}+u_{1} \\
& \dot{y}_{s}=-c y_{s}^{3}+d x_{s} z_{s}+u_{2} \\
& \dot{z}_{s}=f z_{s}-g x_{s} y_{s}+u_{3}
\end{aligned}
$$

where $u_{1}, u_{2}$, and $u_{3}$ are control inputs, and the subscript $s$ denotes "slave." The parameters of systems (3) and (4) are considered unknown. In this paper, a proper control law will be determined such that system (4) can follow the chaotic behavior of system (3) in spite of the lack of knowledge about the parameter values.

\section{Adaptive Stabilization}

In this section, the stabilization problem of system (2) with unknown parameters is considered. First, the ideal case is solved by means of Lyapunov-like stability theory. Next, it is shown as, in the presence of unmodeled dynamics and/or disturbances, the recently deduced control law is not effective anymore. Thus, a modification of such control law must be done to overcome this drawback. 


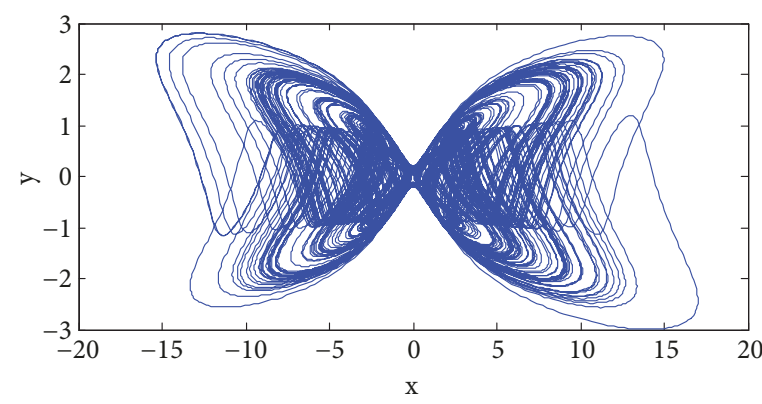

(a)

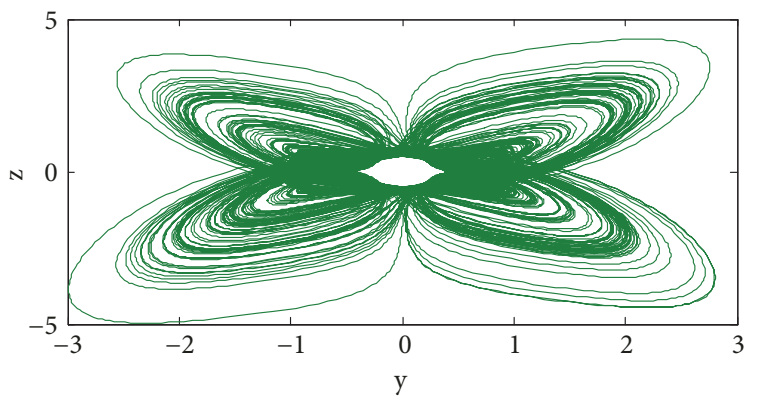

(c)

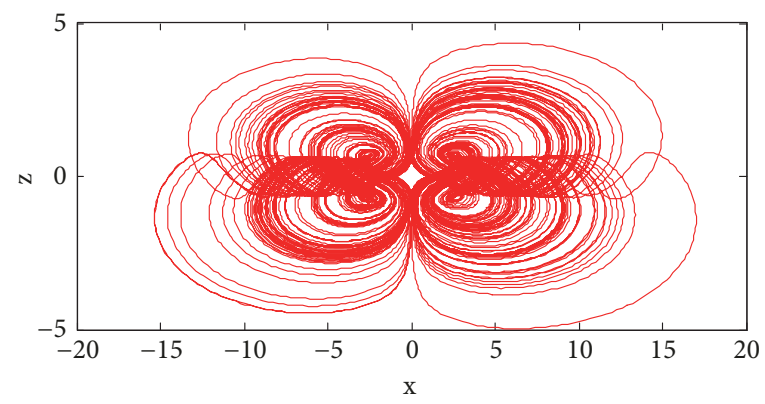

(b)

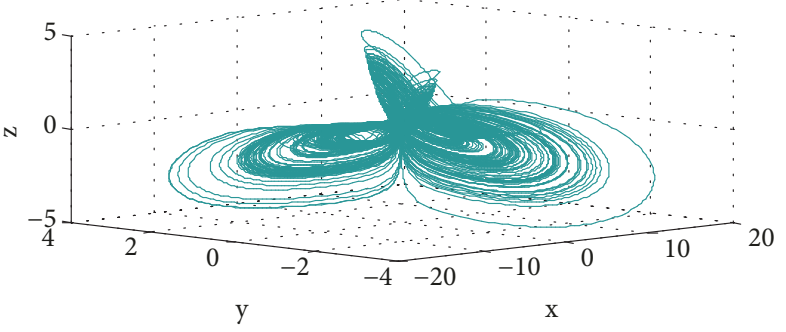

(d)

Figure 1: Four-scroll attractor for Zhang system: (a) $x-y$ phase plane, (b) $x-z$ phase plane, (c) $y-z$ phase plane, and (d) phase space.

3.1. Ideal Case. Given system (2) and considering that the parameters are completely unknown, the following control law can be proposed:

$$
\begin{aligned}
& \mu_{1}=\widehat{a} x-\widehat{b} y z-k_{1} x \\
& \mu_{2}=\widehat{c} y^{3}-\widehat{d} x z-k_{2} y \\
& \mu_{3}=-\widehat{f} z+\widehat{g} x y-k_{3} z
\end{aligned}
$$

where $\widehat{a}, \widehat{b}, \widehat{c}, \widehat{d}, \widehat{f}$, and $\widehat{g}$ are the estimations of unknown constant parameters $a, b, c, d, f$, and $g$, respectively; $k_{1}, k_{2}$, and $k_{3}$ are positive constants selectable by the designer. By substituting (5) into (2), the following closed-loop dynamics is obtained:

$$
\begin{aligned}
& \dot{x}=\widetilde{a} x-\widetilde{b} y z-k_{1} x \\
& \dot{y}=\widetilde{c} y^{3}-\widetilde{d} x z-k_{2} y \\
& \dot{z}=-\tilde{f} z+\widetilde{g} x y-k_{3} z
\end{aligned}
$$

where

$$
\begin{aligned}
& \widetilde{a}:=\widehat{a}-a \\
& \widetilde{b}:=\widehat{b}-b \\
& \widetilde{c}:=\widehat{c}-c \\
& \widetilde{d}:=\widehat{d}-d \\
& \widetilde{f}:=\widehat{f}-f \\
& \widetilde{g}:=\widehat{g}-g
\end{aligned}
$$

Note that, by taking the first time derivative of (7) and as each unknown parameter is constant, we can get

$$
\begin{aligned}
& \dot{\tilde{a}}=\dot{\vec{a}} \\
& \dot{\vec{b}}=\dot{\vec{b}} \\
& \dot{\tilde{c}}=\dot{\vec{c}} \\
& \dot{\tilde{d}}=\dot{\vec{d}} \\
& \dot{\tilde{f}}:=\dot{\vec{f}} \\
& \dot{\tilde{g}}:=\dot{\vec{g}}
\end{aligned}
$$

In order to analyze the stability of (6), the following Lyapunov function candidate is proposed:

$$
\begin{aligned}
& V(x, y, z, \widetilde{a}, \widetilde{b}, \widetilde{c}, \widetilde{d}, \widetilde{f}, \widetilde{g})=\frac{1}{2}\left(x^{2}+y^{2}+z^{2}+\frac{1}{\gamma_{1}} \widetilde{a}^{2}\right. \\
& \left.+\frac{1}{\gamma_{2}} \widetilde{b}^{2}+\frac{1}{\gamma_{3}} \widetilde{c}^{2}+\frac{1}{\gamma_{4}} \widetilde{d}^{2}+\frac{1}{\gamma_{5}} \widetilde{f}^{2}+\frac{1}{\gamma_{6}} \widetilde{g}^{2}\right)
\end{aligned}
$$

where $\gamma_{1}, \gamma_{2}, \gamma_{3}, \gamma_{4}, \gamma_{5}$, and $\gamma_{6}$ are positive constants selectable by the designer. Note that (9) is a positive definite on $\mathbb{R}^{9}$. The first time derivative of (9) can be calculated as

$$
\begin{aligned}
\dot{V}= & x \dot{x}+y \dot{y}+z \dot{z}+\frac{1}{\gamma_{1}} \widetilde{a} \dot{\tilde{a}}+\frac{1}{\gamma_{2}} \widetilde{b} \dot{\vec{b}}+\frac{1}{\gamma_{3}} \tilde{c} \dot{\tilde{c}}+\frac{1}{\gamma_{4}} \widetilde{d} \dot{\tilde{d}} \\
& +\frac{1}{\gamma_{5}} \tilde{f} \dot{\tilde{f}}+\frac{1}{\gamma_{6}} \widetilde{g} \dot{\tilde{g}}
\end{aligned}
$$


By substituting (6) into (10), taking into account (8), and after some operations, it is possible to obtain

$$
\begin{aligned}
\dot{V}= & \left(x^{2}+\frac{1}{\gamma_{1}} \dot{\hat{a}}\right) \tilde{a}+\left(-x y z+\frac{1}{\gamma_{2}} \dot{\hat{b}}\right) \tilde{b} \\
& +\left(y^{4}+\frac{1}{\gamma_{3}} \dot{\hat{c}}\right) \tilde{c}+\left(-x y z+\frac{1}{\gamma_{4}} \dot{\hat{d}}\right) \tilde{d} \\
& +\left(-z^{2}+\frac{1}{\gamma_{5}} \dot{\hat{f}}\right) \tilde{f}+\left(x y z+\frac{1}{\gamma_{6}} \dot{\hat{g}}\right) \tilde{g}-k_{1} x^{2} \\
& -k_{2} y^{2}-k_{3} z^{2}
\end{aligned}
$$

In view of (11), the following update law can be deduced:

$$
\begin{aligned}
& \dot{\hat{a}}=-\gamma_{1} x^{2} \\
& \dot{\hat{b}}=\gamma_{2} x y z \\
& \dot{\hat{c}}=-\gamma_{3} y^{4} \\
& \dot{\hat{d}}=\gamma_{4} x y z \\
& \dot{\hat{f}}=\gamma_{5} z^{2} \\
& \dot{\hat{g}}=-\gamma_{6} x y z
\end{aligned}
$$

If (12) is substituted into (11), then

$$
\dot{V}=-k_{1} x^{2}-k_{2} y^{2}-k_{3} z^{2} \leq 0
$$

Consequently, the first time derivative of $V$ is negative semidefinite. Thus, the asymptotic stability cannot be concluded from the second theorem of Lyapunov and an additional analysis is required. Alternatively, (13) can be expressed as

$$
\frac{d V}{d t} \leq 0
$$

Or

$$
d V \leq-0 d t
$$

By integrating both sides of the last inequality from 0 to $t$, it follows that

$$
\begin{gathered}
\int_{V(0)}^{V(t)} d V \leq-\int_{0}^{t} 0 d t \\
V(t)-V(0) \leq 0
\end{gathered}
$$

$$
V \leq V(0)
$$

and as $V$ is a nonnegative function, then $V \in L_{\infty}$. Consequently, $x, y, z, \widetilde{a}, \widetilde{b}, \widetilde{c}, \widetilde{d}, \widetilde{f}$, and $\widetilde{g}$ belong to $L_{\infty}$. From (7) and as $a, b, c, d, f$, and $g$ are constant parameters, then it can be concluded that $\widehat{a}, \widehat{b}, \widehat{c}, \widehat{d}, \widehat{f}$, and $\widehat{g}$ also belong to $L_{\infty}$. An inspection of (5) and (6) reveals that $\mu_{1}, \mu_{2}, \mu_{3}, \dot{x}, \dot{y}$, and $\dot{z}$ are formed by bounded terms. Thus, $\mu_{1}, \mu_{2}, \mu_{3}, \dot{x}, \dot{y}, \dot{z} \in L_{\infty}$. On other hand, considering the following definition

$$
k:=\min \left(k_{1}, k_{2}, k_{3}\right)
$$

and taking into account (13), it can be established that

$$
d V \leq-k\left(x^{2}+y^{2}+z^{2}\right) d \tau
$$

By integrating both sides of (18) from zero to $t$, it can be obtained that

$$
V(t)-V(0) \leq-k \int_{0}^{t}\left(x^{2}+y^{2}+z^{2}\right) d \tau
$$

or well

$$
\int_{0}^{t}\left(x^{2}+y^{2}+z^{2}\right) d \tau \leq \frac{V(0)-V(t)}{k}
$$

Now, as $V(t)$ is a nonnegative function, the following is true:

$$
\begin{aligned}
V(t) & \geq 0 \Longrightarrow \\
V(t)-V(0) & \geq-V(0) \Longrightarrow \\
V(0)-V(t) & \leq V(0)
\end{aligned}
$$

Substituting (21) into (20) yields

$$
\int_{0}^{t}\left(x^{2}+y^{2}+z^{2}\right) d \tau \leq \frac{V(0)}{k}
$$

By taking the limit as $t \longrightarrow \infty$ of both sides of the last inequality, finally, it can be obtained that

$$
\int_{0}^{\infty}\left(x^{2}+y^{2}+z^{2}\right) d \tau \leq \frac{V(0)}{k}
$$

This means that $x, y, z \in L_{2}$. As $x, y, z \in L_{2} \cap L_{\infty}$ and $\dot{x}, \dot{y}, \dot{z} \in L_{\infty}$ then it can be concluded from Barbalat's lemma [82] that $x, y$, and $z$ converge asymptotically to zero. Hence, the following result has been proven:

Lemma 1. If the control law (5) with the learning law (12) is applied to Zhang system (2), then

(a) the states, the estimations of the parameters, and the control signal are bounded, that is,

$$
x, y, z, \widehat{a}, \widehat{b}, \widehat{c}, \widehat{d}, \widehat{f}, \widehat{g}, \mu_{1}, \mu_{2}, \mu_{3} \in L_{\infty}
$$

(b) the states $x, y$, and $z$ converge globally and asymptotically to zero.

Corollary 2. As the estimations of the parameters $\widehat{a}, \widehat{b}, \widehat{c}, \widehat{d}, \widehat{f}, \widehat{g}$ are bounded and besides $\lim _{t \rightarrow \infty} \dot{\hat{a}}=$ $\lim _{t \rightarrow \infty} \dot{\hat{\vec{b}}}=\lim _{t \rightarrow \infty} \dot{\hat{c}}=\lim _{t \rightarrow \infty} \dot{\hat{d}}=\lim _{t \rightarrow \infty} \dot{\hat{f}}=$ $\lim _{t \rightarrow \infty} \dot{\widehat{g}}=0$ then each estimation converges to a constant value.

The performance of the control law (5) can be tested by simulation. In order to achieve this objective, first system (2) is implemented in Simulink ${ }^{\circledR}$ with the parameter values provided in Section 2 and with the initial condition $x(0)=$ $5, y(0)=-5$, and $z(0)=7$. The loop is closed with the control law (5) and the learning law (12). For simplicity, the 
gains of the control law are selected as $k_{1}=1, k_{2}=1$, and $k_{3}=1$ and the parameters of the learning law are selected as $\gamma_{1}=\gamma_{2}=\gamma_{3}=\gamma_{4}=\gamma_{5}=\gamma_{6}=1$ with the initial condition $\widehat{a}(0)=\widehat{b}(0)=\widehat{c}(0)=\widehat{d}(0)=\widehat{f}(0)=\widehat{g}(0)=0$. The simulation is carried out by means of Runge-Kutta method (ode4) with a fixed-step size of 0.0001 . The results of the simulation process are presented in Figures 2, 3, and 4.

In Figure 2, the stabilization process for the three states of system (2) is illustrated for the first 1.5 seconds $(s)$. It can be appreciated as the stabilization is attained in less than $1 \mathrm{~s}$. In Figure 3, the estimations of the constant parameters $a, c$, and $f$ are shown. According to Corollary 2, each estimation tends to a constant value. However, it should be mentioned that this constant value is not necessarily the current value of the corresponding parameter. Finally, the control law (5) is depicted in Figure 4. Once the stabilization is attained, each control input is equal to zero.

3.2. Nonideal Case. Now, let us consider the more realistic case when unmodeled dynamics and/or disturbances are present into system (2). This effect can be described by means of the terms $\omega_{1}, \omega_{2}$, and $\omega_{3}$ in the following way:

$$
\begin{aligned}
& \dot{x}=-a x+b y z+\mu_{1}+\omega_{1} \\
& \dot{y}=-c y^{3}+d x z+\mu_{2}+\omega_{2} \\
& \dot{z}=f z-g x y+\mu_{3}+\omega_{3}
\end{aligned}
$$

Assumption 3. Although the terms $\omega_{1}, \omega_{2}$, and $\omega_{3}$ represent unknown unmodeled dynamics and/or disturbance, a bound $\bar{\omega}_{1}, \bar{\omega}_{2}$, and $\bar{\omega}_{3}$ for each term, respectively, is a priori known, that is, $\left|\omega_{1}\right| \leq \bar{\omega}_{1},\left|\omega_{2}\right| \leq \bar{\omega}_{2}$, and $\left|\omega_{3}\right| \leq \bar{\omega}_{3}$.

Under the existence of unmodeled dynamics and/or disturbance, the control law (5) presented in Lemma 1 is not effective anymore. In order to illustrate this point, consider that $\omega_{1}, \omega_{2}$, and $\omega_{3}$ are given by $\omega_{1}=\omega_{2}=\omega_{3}=5 \sin (10 t)$. On these new conditions, the simulation of system (25) under the control law (5) with the learning law (12) produces the results exhibited in Figure 5. Apparently, the main performance deterioration is the presence of a sinusoidal signal of about 0.5 amplitude in steady-state. However, a simulation during $5000 s$ shows how now the estimations do not converge to a constant value (see Figure 6). This process is known as drift and ultimately causes instability of the system. Consequently, the control law and the learning law must be modified to overcome this problem.

Following the approach presented in [83], in this work, the control law (5) is modified by adding a robustifying term, which consists of a saturation function:

$$
\begin{aligned}
& \mu_{1}=\widehat{a} x-\widehat{b} y z-k_{1} x-k_{1}^{*} \operatorname{sat}\left(\frac{x}{\varepsilon_{x}}\right) \\
& \mu_{2}=\widehat{c} y^{3}-\widehat{d} x z-k_{2} y-k_{2}^{*} \operatorname{sat}\left(\frac{y}{\varepsilon_{y}}\right) \\
& \mu_{3}=-\widehat{f} z+\widehat{g} x y-k_{3} z-k_{3}^{*} \operatorname{sat}\left(\frac{z}{\varepsilon_{z}}\right)
\end{aligned}
$$

where $k_{1}^{*}, k_{2}^{*}, k_{3}^{*}$ are positive constants, $\varepsilon_{x}, \varepsilon_{y}$, and $\varepsilon_{z}$ are small positive constants, which can be selected by the designer and sat $(\cdot)$ represents a saturation function depicted by

$$
\text { sat }(\xi)= \begin{cases}1 & \text { for } \xi \geq 1 \\ \xi & \text { for }-1<\xi<1 \\ -1 & \text { for } \xi \leq-1\end{cases}
$$

Substituting (26) into (25) and after some operations, the close-loop dynamics is now given by

$$
\begin{aligned}
& \dot{x}=\tilde{a} x-\tilde{b} y z-k_{1} x-k_{1}^{*} \text { sat }\left(\frac{x}{\varepsilon_{x}}\right)+\omega_{1} \\
& \dot{y}=\tilde{c} y^{3}-\tilde{d} x z-k_{2} y-k_{2}^{*} \text { sat }\left(\frac{y}{\varepsilon_{y}}\right)+\omega_{2} \\
& \dot{z}=-\tilde{f} z+\tilde{g} x y-k_{3} z-k_{3}^{*} \text { sat }\left(\frac{z}{\varepsilon_{z}}\right)+\omega_{3}
\end{aligned}
$$

where $\tilde{a}, \widetilde{b}, \widetilde{c}, \widetilde{d}, \widetilde{f}$, and $\widetilde{g}$ are defined as in (7). In order to analyze the close-loop dynamics (28), the following Lyapunov function candidate is proposed:

$$
\begin{gathered}
V\left(x_{\varepsilon_{x}}, y_{\varepsilon_{y}}, z_{\varepsilon_{z}}, \tilde{a}, \tilde{b}, \widetilde{c}, \tilde{d}, \tilde{f}, \tilde{g}\right):=\frac{1}{2}\left(x_{\varepsilon_{x}}^{2}+y_{\varepsilon_{y}}^{2}+z_{\varepsilon_{z}}^{2}\right. \\
\left.+\frac{1}{\gamma_{1}} \widetilde{a}^{2}+\frac{1}{\gamma_{2}} \widetilde{b}^{2}+\frac{1}{\gamma_{3}} \widetilde{c}^{2}+\frac{1}{\gamma_{4}} \widetilde{d}^{2}+\frac{1}{\gamma_{5}} \widetilde{f}^{2}+\frac{1}{\gamma_{6}} \widetilde{g}^{2}\right)
\end{gathered}
$$

where $\gamma_{1}, \gamma_{2}, \gamma_{3}, \gamma_{4}, \gamma_{5}$, and $\gamma_{6}$ are positive constants and

$$
\begin{aligned}
& x_{\varepsilon_{x}}:=x-\varepsilon_{x} \text { sat }\left(\frac{x}{\varepsilon_{x}}\right) \\
& y_{\varepsilon_{y}}:=y-\varepsilon_{y} \text { sat }\left(\frac{y}{\varepsilon_{y}}\right) \\
& z_{\varepsilon_{z}}:=z-\varepsilon_{z} \text { sat }\left(\frac{z}{\varepsilon_{z}}\right)
\end{aligned}
$$

The first time derivative of (29) is

$$
\begin{aligned}
\dot{V}= & x_{\varepsilon_{x}} \dot{x}_{\varepsilon_{x}}+y_{\varepsilon_{y}} \dot{y}_{\varepsilon_{y}}+z_{\varepsilon_{z}} \dot{z}_{\varepsilon_{z}}+\frac{1}{\gamma_{1}} \tilde{a} \dot{\tilde{a}}+\frac{1}{\gamma_{2}} \tilde{b} \dot{\widetilde{b}}+\frac{1}{\gamma_{3}} \tilde{c} \dot{\tilde{c}} \\
& +\frac{1}{\gamma_{4}} \tilde{d} \dot{\tilde{d}}+\frac{1}{\gamma_{5}} \tilde{f} \dot{\tilde{f}}+\frac{1}{\gamma_{6}} \tilde{g} \dot{\tilde{g}}
\end{aligned}
$$

It can be proven that

$$
\begin{aligned}
& x_{\varepsilon_{x}} \dot{x}_{\varepsilon_{x}}=x_{\varepsilon_{x}} \dot{x} \\
& y_{\varepsilon_{y}} \dot{y}_{\varepsilon_{y}}=y_{\varepsilon_{y}} \dot{y} \\
& z_{\varepsilon_{z}} \dot{z}_{\varepsilon_{z}}=z_{\varepsilon_{z}} \dot{z}
\end{aligned}
$$

Taking into account the previous fact and according to (8), (31) becomes

$$
\begin{aligned}
\dot{V}= & x_{\varepsilon_{x}} \dot{x}+y_{\varepsilon_{y}} \dot{y}+z_{\varepsilon_{z}} \dot{z}+\frac{1}{\gamma_{1}} \tilde{a} \dot{\hat{a}}+\frac{1}{\gamma_{2}} \widetilde{b} \dot{\hat{b}}+\frac{1}{\gamma_{3}} \tilde{c} \dot{\vec{c}} \\
& +\frac{1}{\gamma_{4}} \tilde{d} \dot{\hat{d}}+\frac{1}{\gamma_{5}} \tilde{f} \dot{\hat{f}}+\frac{1}{\gamma_{6}} \tilde{g} \dot{\hat{g}}
\end{aligned}
$$




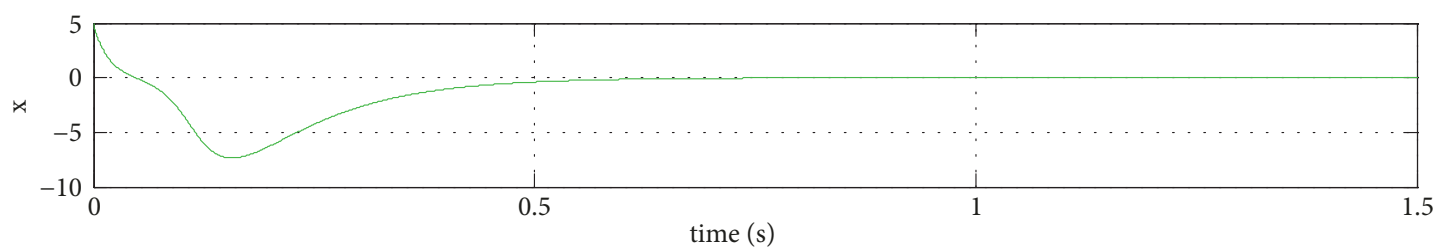

(a)

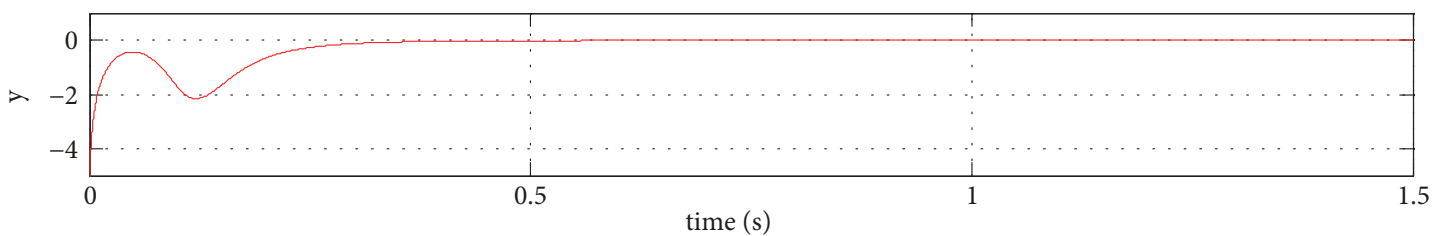

(b)

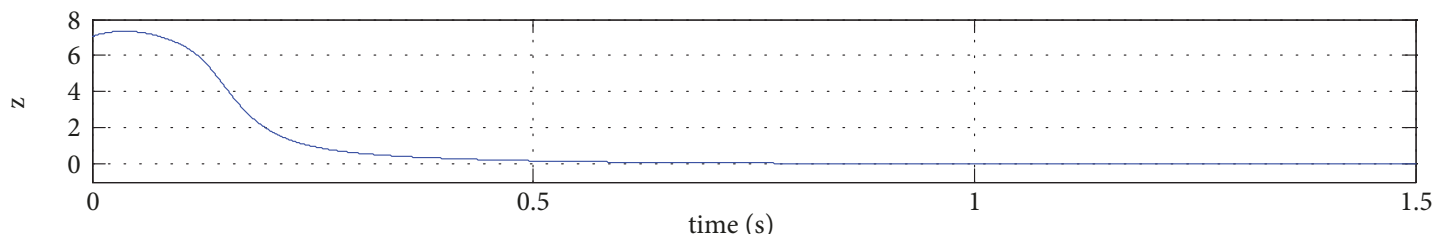

(c)

FigURE 2: Adaptive stabilization for (a) state $x$, (b) state $y$, and (c) state $z$.

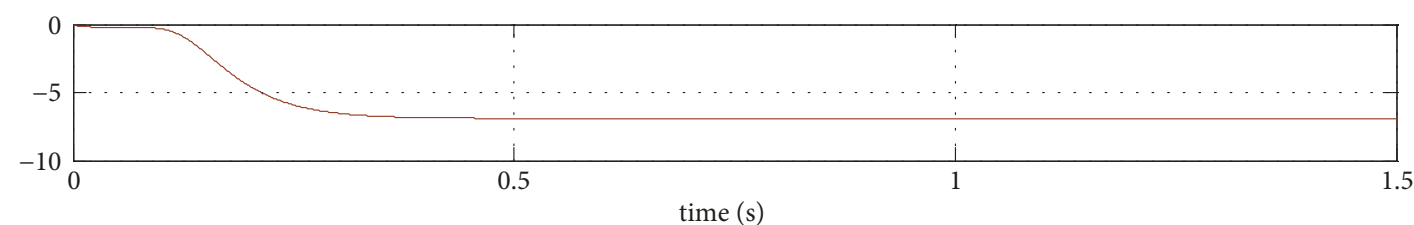

(a)

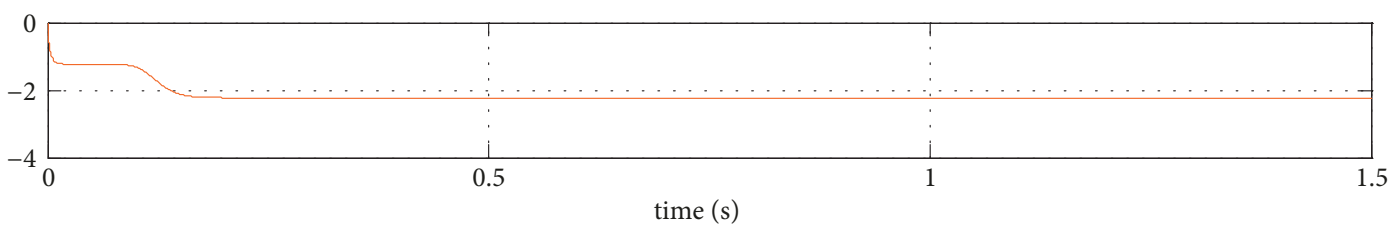

(b)

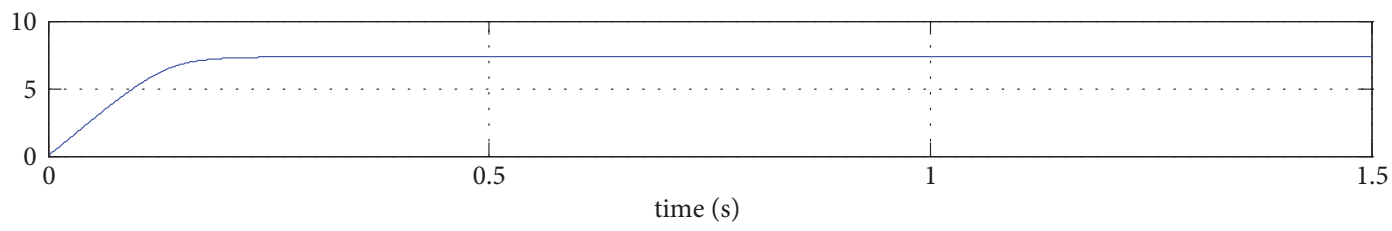

(c)

FIGURE 3: Estimations of the unknown parameters: (a) $\widehat{a}$, (b) $\widehat{c}$, and (c) $\widehat{f}$.

By substituting (28) into (33), and after accomplishing the corresponding operations and grouping like terms, the following is obtained:

$$
\dot{V}=\widetilde{a}\left(x_{\varepsilon_{x}} x+\frac{1}{\gamma_{1}} \dot{\hat{a}}\right)+\widetilde{b}\left(-x_{\varepsilon_{x}} y z+\frac{1}{\gamma_{2}} \dot{\hat{b}}\right)
$$

$$
\begin{aligned}
& +\widetilde{c}\left(y_{\varepsilon_{y}} y^{3}+\frac{1}{\gamma_{3}} \dot{\hat{c}}\right)+\tilde{d}\left(-y_{\varepsilon_{y}} x z+\frac{1}{\gamma_{4}} \dot{\hat{d}}\right) \\
& +\tilde{f}\left(-z_{\varepsilon_{z}} z+\frac{1}{\gamma_{5}} \dot{\hat{f}}\right)+\widetilde{g}\left(z_{\varepsilon_{z}} x y+\frac{1}{\gamma_{6}} \dot{\hat{g}}\right) \\
& -k_{1} x_{\varepsilon_{x}} x-k_{1}^{*} x_{\varepsilon_{x}} \operatorname{sat}\left(\frac{x}{\varepsilon_{x}}\right)+x_{\varepsilon_{x}} \omega_{1}-k_{2} y_{\varepsilon_{y}} y
\end{aligned}
$$




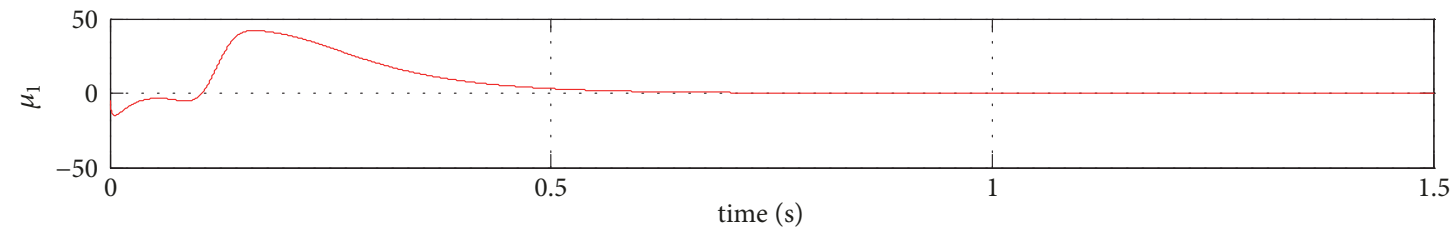

(a)

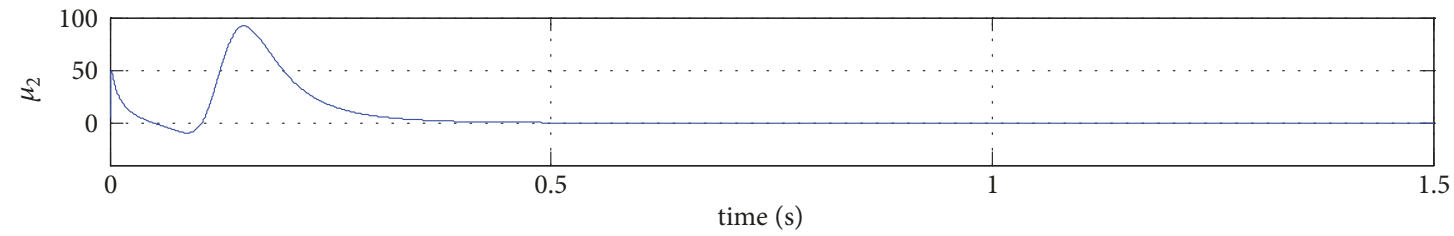

(b)

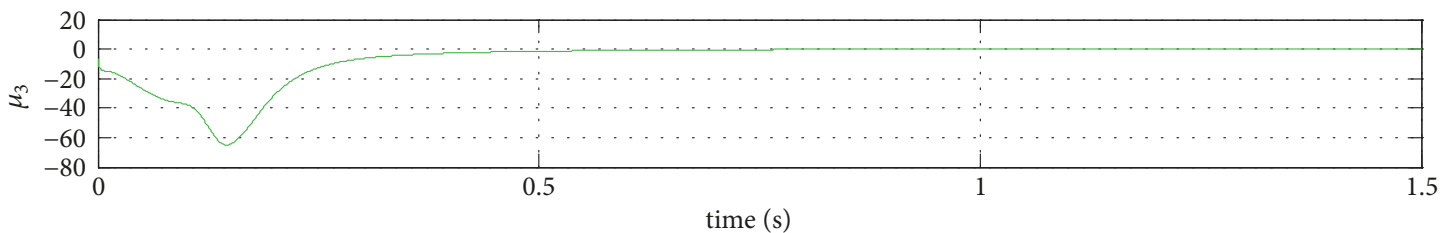

(c)

FiguRE 4: Control law: (a) $\mu_{1}$, (b) $\mu_{2}$, and (c) $\mu_{3}$.

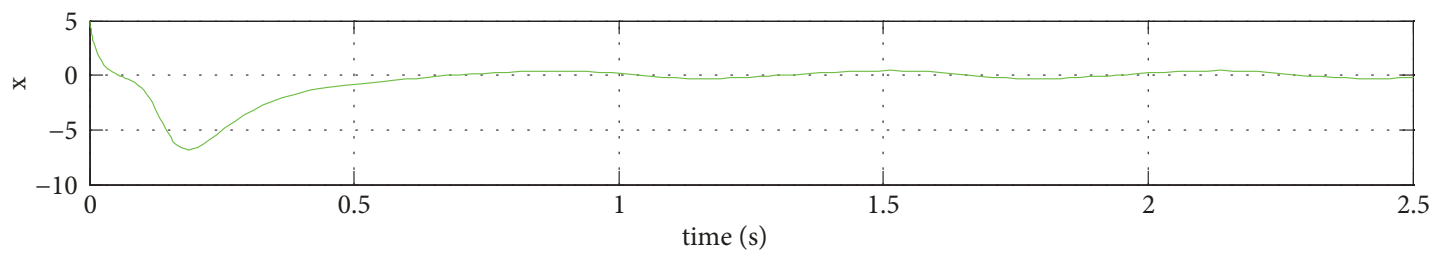

(a)

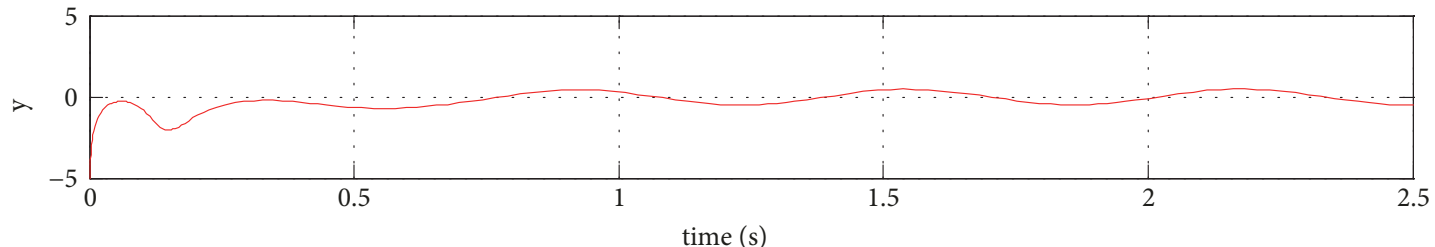

(b)

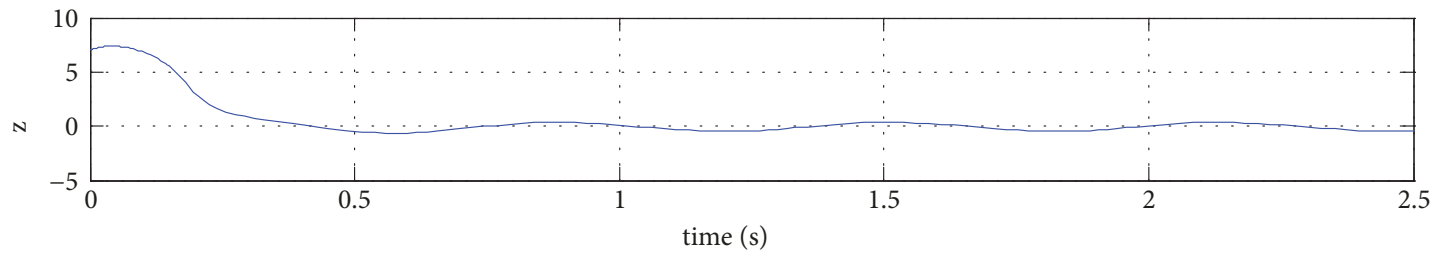

(c)

FIGURE 5: Application of the control law (5) for adaptive stabilization of nonideal Zhang system (25): (a) state $x$, (b) state $y$, and (c) state $z$.

$$
\begin{aligned}
& -k_{2}^{*} y_{\varepsilon_{y}} \text { sat }\left(\frac{y}{\varepsilon_{y}}\right)+y_{\varepsilon_{y}} \omega_{2}-k_{3} z_{\varepsilon_{z}} z \\
& -k_{3}^{*} z_{\varepsilon_{z}} \operatorname{sat}\left(\frac{z}{\varepsilon_{z}}\right)+z_{\varepsilon_{z}} \omega_{3}
\end{aligned}
$$

In view of (34), the following update law can be deduced:

$$
\begin{aligned}
& \dot{\hat{a}}=-\gamma_{1} x_{\varepsilon_{x}} x \\
& \dot{\hat{b}}=\gamma_{2} x_{\varepsilon_{x}} y z
\end{aligned}
$$




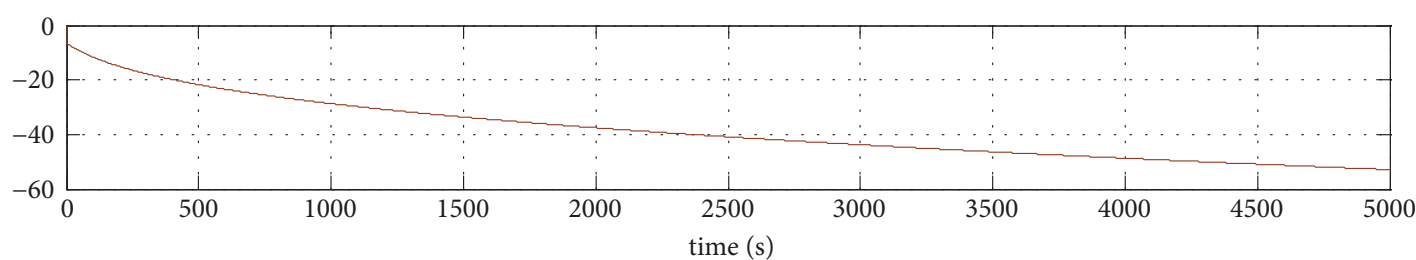

(a)

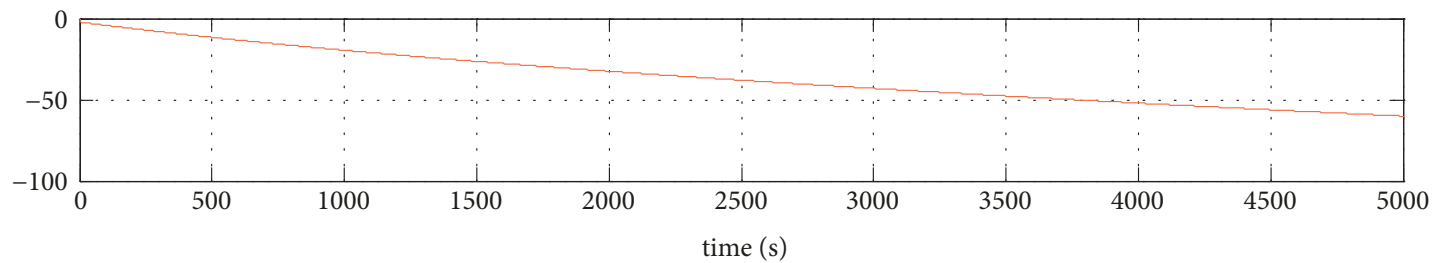

(b)

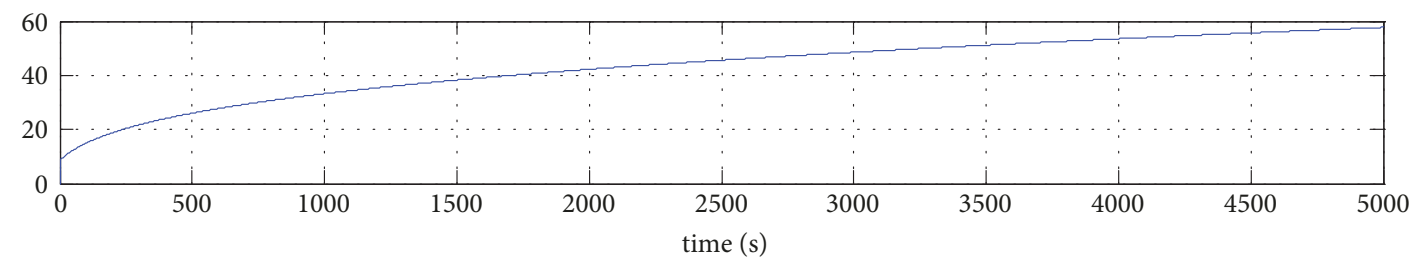

(c)

FIGURE 6: Drift of the unknown parameter estimations: (a) $\widehat{a}$, (b) $\widehat{c}$, and (c) $\widehat{f}$.

$$
\begin{aligned}
& \dot{\hat{c}}=-\gamma_{3} y_{\varepsilon_{y}} y^{3} \\
& \dot{\hat{d}}=\gamma_{4} y_{\varepsilon_{y}} x z \\
& \dot{\hat{f}}=\gamma_{5} z_{\varepsilon_{z}} z \\
& \dot{\hat{g}}=-\gamma_{6} z_{\varepsilon_{z}} x y
\end{aligned}
$$

If (35) is substituted into (34), then the first time derivative of $V$ becomes

$$
\begin{aligned}
\dot{V}= & -k_{1} x_{\varepsilon_{x}} x-k_{1}^{*} x_{\varepsilon_{x}} \text { sat }\left(\frac{x}{\varepsilon_{x}}\right)+x_{\varepsilon_{x}} \omega_{1}-k_{2} y_{\varepsilon_{y}} y \\
& -k_{2}^{*} y_{\varepsilon_{y}} \operatorname{sat}\left(\frac{y}{\varepsilon_{y}}\right)+y_{\varepsilon_{y}} \omega_{2}-k_{3} z_{\varepsilon_{z}} z \\
& -k_{3}^{*} z_{\varepsilon_{z}} \text { sat }\left(\frac{z}{\varepsilon_{z}}\right)+z_{\varepsilon_{z}} \omega_{3}
\end{aligned}
$$

Now, from (30)

$$
\begin{aligned}
& x=x_{\varepsilon_{x}}+\varepsilon_{x} \operatorname{sat}\left(\frac{x}{\varepsilon_{x}}\right) \\
& y=y_{\varepsilon_{y}}+\varepsilon_{y} \operatorname{sat}\left(\frac{y}{\varepsilon_{y}}\right) \\
& z=z_{\varepsilon_{z}}+\varepsilon_{z} \operatorname{sat}\left(\frac{z}{\varepsilon_{z}}\right)
\end{aligned}
$$

Additionally, from Assumption 3, the terms $x_{\varepsilon_{x}} \omega_{1}, y_{\varepsilon_{y}} \omega_{2}$, and $z_{\varepsilon_{z}} \omega_{3}$ can be bounded as

$$
\begin{aligned}
& x_{\varepsilon_{x}} \omega_{1} \leq\left|x_{\varepsilon_{x}} \omega_{1}\right| \leq\left|x_{\varepsilon_{x}}\right| \bar{\omega}_{1} \\
& y_{\varepsilon_{y}} \omega_{2} \leq\left|y_{\varepsilon_{y}} \omega_{2}\right| \leq\left|y_{\varepsilon_{y}}\right| \bar{\omega}_{2} \\
& z_{\varepsilon_{z}} \omega_{3} \leq\left|z_{\varepsilon_{z}} \omega_{3}\right| \leq\left|z_{\varepsilon_{z}}\right| \bar{\omega}_{3}
\end{aligned}
$$

Taking into account (37) and (38), and next grouping like terms, (36) becomes

$$
\begin{aligned}
\dot{V} \leq & -k_{1} x_{\varepsilon_{x}}^{2}-\left(k_{1} \varepsilon_{x}+k_{1}^{*}\right) x_{\varepsilon_{x}} \text { sat }\left(\frac{x}{\varepsilon_{x}}\right)+\left|x_{\varepsilon_{x}}\right| \bar{\omega}_{1} \\
& -k_{2} y_{\varepsilon_{y}}^{2}-\left(k_{2} \varepsilon_{y}+k_{2}^{*}\right) y_{\varepsilon_{y}} \text { sat }\left(\frac{y}{\varepsilon_{y}}\right)+\left|y_{\varepsilon_{y}}\right| \bar{\omega}_{2} \\
& -k_{3} z_{\varepsilon_{z}}^{2}-\left(k_{3} \varepsilon_{z}+k_{3}^{*}\right) z_{\varepsilon_{z}} \text { sat }\left(\frac{z}{\varepsilon_{z}}\right)+\left|z_{\varepsilon_{z}}\right| \bar{\omega}_{3}
\end{aligned}
$$

it can be shown that

$$
\begin{aligned}
& x_{\varepsilon_{x}} \text { sat }\left(\frac{x}{\varepsilon_{x}}\right)=\left|x_{\varepsilon_{x}}\right| \\
& y_{\varepsilon_{y}} \text { sat }\left(\frac{y}{\varepsilon_{y}}\right)=\left|y_{\varepsilon_{y}}\right| \\
& z_{\varepsilon_{z}} \text { sat }\left(\frac{z}{\varepsilon_{z}}\right)=\left|z_{\varepsilon_{z}}\right|
\end{aligned}
$$


Substituting (40) into (39) and grouping like terms, we can get

$$
\begin{aligned}
\dot{V} \leq & -k_{1} x_{\varepsilon_{x}}^{2}-\left(k_{1} \varepsilon_{x}+k_{1}^{*}-\bar{\omega}_{1}\right)\left|x_{\varepsilon_{x}}\right|-k_{2} y_{\varepsilon_{y}}^{2} \\
& -\left(k_{2} \varepsilon_{y}+k_{2}^{*}-\bar{\omega}_{2}\right)\left|y_{\varepsilon_{y}}\right|-k_{3} z_{\varepsilon_{z}}^{2} \\
& -\left(k_{3} \varepsilon_{z}+k_{3}^{*}-\bar{\omega}_{3}\right)\left|z_{\varepsilon_{z}}\right|
\end{aligned}
$$

if the constants $k_{1}, k_{1}^{*}, \varepsilon_{x}, k_{2}, k_{2}^{*}, \varepsilon_{y}, k_{3}, k_{3}^{*}$, and $\varepsilon_{z}$ are selected in such a way that the following inequality is satisfied

$$
\begin{aligned}
& k_{1} \varepsilon_{x}+k_{1}^{*}>\bar{\omega}_{1} \\
& k_{2} \varepsilon_{y}+k_{2}^{*}>\bar{\omega}_{2} \\
& k_{3} \varepsilon_{z}+k_{3}^{*}>\bar{\omega}_{3}
\end{aligned}
$$

then, from (41), it can be seen that

$$
\dot{V} \leq-k_{1} x_{\varepsilon_{x}}^{2}-k_{2} y_{\varepsilon_{y}}^{2}-k_{3} z_{\varepsilon_{z}}^{2}
$$

That means that $\dot{V} \leq 0$ which implies that $V \leq V(0)$ and $x_{\varepsilon_{x}}, y_{\varepsilon_{y}}, z_{\varepsilon_{z}}, \widehat{a}, \widehat{b}, \widehat{c}, \widehat{d}, \hat{f}, \widehat{g} \in L_{\infty}$. From the boundedness of $x_{\varepsilon_{x}}, y_{\varepsilon_{y}}, z_{\varepsilon_{z}}$ and from (37) the following can be concluded: $x, y, z \in L_{\infty}$. As the control signal (26) and the first time derivative of $x_{\varepsilon_{x}}, y_{\varepsilon_{y}}, z_{\varepsilon_{z}}$ are formed by bounded terms, then $\mu_{1}, \mu_{2}, \mu_{3}, \dot{x}_{\varepsilon_{x}}, \dot{y}_{\varepsilon_{y}}, \dot{z}_{\varepsilon_{z}} \in L_{\infty}$. From (17), (43) can be expressed as

$$
\dot{V} \leq-k\left(x_{\varepsilon_{x}}^{2}+y_{\varepsilon_{y}}^{2}+z_{\varepsilon_{z}}^{2}\right)
$$

Due to an argument similar to Section 3.1, it can be shown that

$$
\int_{0}^{\infty}\left(x_{\varepsilon_{x}}^{2}+y_{\varepsilon_{y}}^{2}+z_{\varepsilon_{z}}^{2}\right) d \tau \leq \frac{V(0)}{k}
$$

That is, $x_{\varepsilon_{x}}, y_{\varepsilon_{y}}, z_{\varepsilon_{z}} \in L_{2}$. As $x_{\varepsilon_{x}}, y_{\varepsilon_{y}}, z_{\varepsilon_{z}} \in L_{2} \cap L_{\infty}$ and $\dot{x}_{\varepsilon_{x}}, \dot{y}_{\varepsilon_{y}}, \dot{z}_{\varepsilon_{z}} \in L_{\infty}$, from the Barbalat's lemma the asymptotic convergence to zero of $x_{\varepsilon_{x}}, y_{\varepsilon_{y}}, z_{\varepsilon_{z}}$ is guaranteed. Taking into account this fact and from (37), finally we can conclude that $x, y, z$ converge asymptotically to a zone bounded by $\varepsilon_{x}, \varepsilon_{y}$, and $\varepsilon_{z}$, respectively. Thus, the following theorem has been demonstrated:

Theorem 4. If Assumption 3 is satisfied, the constants $k_{1}, k_{1}^{*}, \varepsilon_{x}, k_{2}, k_{2}^{*}, \varepsilon_{y}, k_{3}, k_{3}^{*}$, and $\varepsilon_{z}$ are selected in such a way that inequality (42) is verified, and control law (26) with learning law (35) is applied to uncertain Zhang system (25), then

(a) $x_{\varepsilon_{x}}, y_{\varepsilon_{y}}, z_{\varepsilon_{z}}, x, y, z, \widehat{a}, \widehat{b}, \widehat{c}, \widehat{d}, \widehat{f}, \widehat{g}, \mu_{1}, \mu_{2}, \mu_{3} \in L_{\infty}$

(b) the states $x, y$, and $z$ converge asymptotically to a region around zero bounded by $\varepsilon_{x}, \varepsilon_{y}$, and $\varepsilon_{z}$, respectively.

It is worth mentioning that Theorem 4 only guarantees the asymptotic convergence to a bounded zone around zero for $x, y$, and $z$. Thus, rigorously speaking, the convergence time could be infinite and consequently this result does not provide any procedure to determine it. Fortunately, as it will be seen later, numerical simulations show that this convergence time is finite. Although it is not possible to establish any analytical relationship, it can be mentioned that, in general, when the gains $k_{1}, k_{2}, k_{3}, k_{1}^{*}, k_{2}^{*}, k_{3}^{*}$ are increased, then the convergence time is decreased. However, it should be taken into account that the magnitude of the corresponding control signals $\mu_{1}, \mu_{2}, \mu_{3}$ can reach excessively large values. Therefore, a trade-off must be established. With respect to the parameters of the learning law (35), $\gamma_{1}, \gamma_{2}, \gamma_{3}, \gamma_{4}, \gamma_{5}, \gamma_{6}$, and the initial conditions of the estimations of the unknown parameters $\widehat{a}(0), \widehat{b}(0), \widehat{c}(0), \widehat{d}(0), \widehat{f}(0), \widehat{g}(0)$, there is not a completely clear relationship between the effect of these parameters and the performance of the closed-loop system. Due to this reason, they should be selected by a trial and error process. Given the large number of parameters to be tuned, in order to avoid spending excessive time, this process should be stopped when a set of values produce an acceptable performance (although not necessarily the best one). Thus, for simplicity, the parameters of the control law (26) and the learning law (35) are selected here as $\varepsilon_{x}=\varepsilon_{y}=\varepsilon_{z}=0.001$, $k_{1}=k_{2}=k_{3}=1, k_{1}^{*}=k_{2}^{*}=k_{3}^{*}=7, \gamma_{1}=\gamma_{2}=\gamma_{3}=$ $\gamma_{4}=\gamma_{5}=\gamma_{6}=1$, and $\widehat{a}(0)=\widehat{b}(0)=\widehat{c}(0)=\widehat{d}(0)=$ $\widehat{f}(0)=\hat{g}(0)=0$. Again, the initial condition for uncertain Zhang system (25) is equal to $x(0)=5, y(0)=-5, z(0)=7$ and $\omega_{1}=\omega_{2}=\omega_{3}=5 \sin (10 t)$. The corresponding results are presented in Figures 7, 8, and 9. As can be appreciated from Figure 7, the stabilization is attained in less than 0.5 $s$. Although it is not possible to obtain the convergence to zero due to the presence the unmodeled dynamics and/or disturbances, it is possible to guarantee the convergence to a bounded zone. This convergence process is shown in Figure 8. As can be seen, the residual sinusoidal signal has now an amplitude less than 0.001. Finally, it is shown in Figure 9 as the estimations converge to a constant value.

\section{Adaptive Synchronization}

The synchronization of two systems can be considered as the generalization of the stabilization problem previously studied in Section 3. Consequently, the theory learned in that section will be applied in terms of the dynamics of the synchronization error. Again both cases will be considered: (a) ideal case and (b) presence of unmodeled dynamics and/or disturbances.

4.1. Ideal Case. The synchronization error can be defined as

$$
\begin{aligned}
& e_{1}:=x_{s}-x_{m} \\
& e_{2}:=y_{s}-y_{m} \\
& e_{3}:=z_{s}-z_{m}
\end{aligned}
$$

Mathematically, the problem of the synchronization consists of finding a proper control law such that $\lim _{t \rightarrow \infty} e_{1}=$ $\lim _{t \rightarrow \infty} e_{2}=\lim _{t \rightarrow \infty} e_{3}=0$. In order to realize this objective, 


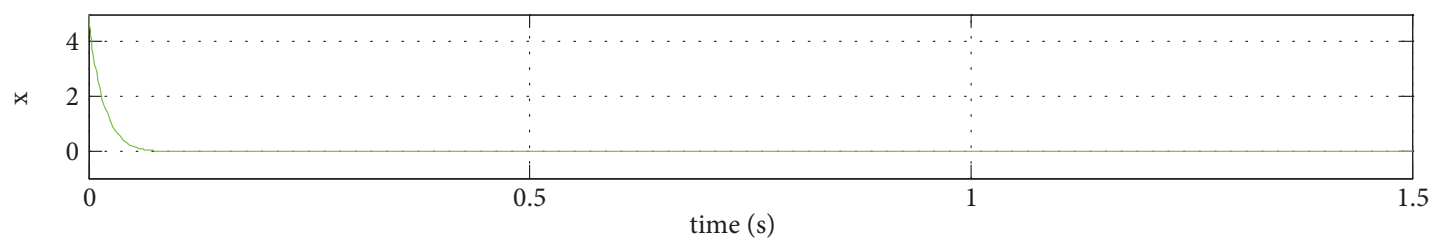

(a)

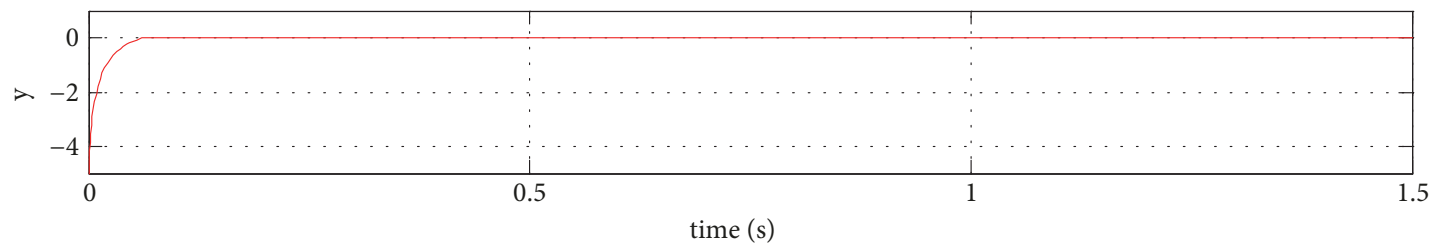

(b)

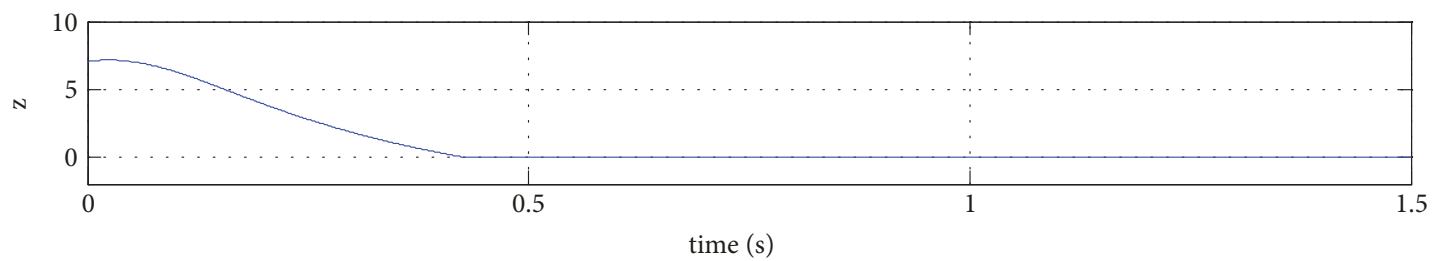

(c)

Figure 7: Robust adaptive stabilization for: (a) state $x$, (b) state $y$, and (c) state $z$.

first, the dynamics of the synchronization error must be determined. This can be done by differentiating (46) and substituting (3) and (4) into the corresponding expression, that is,

$$
\begin{aligned}
\dot{e}_{1} & =\dot{x}_{s}-\dot{x}_{m}=-a x_{s}+b y_{s} z_{s}+u_{1}+a x_{m}-b y_{m} z_{m} \\
& =-a e_{1}+b\left(y_{s} z_{s}-y_{m} z_{m}\right)+u_{1} \\
\dot{e}_{2} & =\dot{y}_{s}-\dot{y}_{m}=-c y_{s}^{3}+d x_{s} z_{s}+u_{2}+c y_{m}^{3}-d x_{m} z_{m} \\
& =-c\left(y_{s}^{3}-y_{m}^{3}\right)+d\left(x_{s} z_{s}-x_{m} z_{m}\right)+u_{2} \\
\dot{e}_{3} & =\dot{z}_{s}-\dot{z}_{m}=f z_{s}-g x_{s} y_{s}+u_{3}-f z_{m}+g x_{m} y_{m} \\
& =f e_{3}-g\left(x_{s} y_{s}-x_{m} y_{m}\right)+u_{3}
\end{aligned}
$$

Once the dynamics of the synchronization error (47) has been obtained, the following control law can be proposed:

$$
\begin{aligned}
& u_{1}=\widehat{a} e_{1}-\widehat{b}\left(y_{s} z_{s}-y_{m} z_{m}\right)-k_{1} e_{1} \\
& u_{2}=\widehat{c}\left(y_{s}^{3}-y_{m}^{3}\right)-\widehat{d}\left(x_{s} z_{s}-x_{m} z_{m}\right)-k_{2} e_{2} \\
& u_{3}=-\widehat{f} e_{3}+\widehat{g}\left(x_{s} y_{s}-x_{m} y_{m}\right)-k_{3} e_{3}
\end{aligned}
$$

where $\widehat{a}, \widehat{b}, \widehat{c}, \widehat{d}, \widehat{f}$, and $\widehat{g}$ are estimations of the unknown constant parameters $a, b, c, d, f$, and $g$, respectively; $k_{1}$, $k_{2}$, and $k_{3}$ are positive constant gains selectable by the designer. By substituting (48) into (47), the closed-loop synchronization error dynamics is obtained as

$$
\begin{aligned}
& \dot{e}_{1}=\widetilde{a} e_{1}-\widetilde{b}\left(y_{s} z_{s}-y_{m} z_{m}\right)-k_{1} e_{1} \\
& \dot{e}_{2}=\widetilde{c}\left(y_{s}^{3}-y_{m}^{3}\right)-\tilde{d}\left(x_{s} z_{s}-x_{m} z_{m}\right)-k_{2} e_{2} \\
& \dot{e}_{3}=-\widetilde{f} e_{3}+\widetilde{g}\left(x_{s} y_{s}-x_{m} y_{m}\right)-k_{3} e_{3}
\end{aligned}
$$

where $\tilde{a}, \tilde{b}, \tilde{c}, \tilde{d}, \tilde{f}$, and $\tilde{g}$ are defined as in (7).

The closed-loop dynamics (49) must be analysed. For this purpose, the following Lyapunov function candidate is proposed:

$$
\begin{aligned}
& V\left(e_{1}, e_{2}, e_{3}, \tilde{a}, \tilde{b}, \tilde{c}, \tilde{d}, \tilde{f}, \tilde{g}\right):=\frac{1}{2}\left(e_{1}^{2}+e_{2}^{2}+e_{3}^{2}+\frac{1}{\gamma_{1}} \tilde{a}^{2}\right. \\
& \left.+\frac{1}{\gamma_{2}} \widetilde{b}^{2}+\frac{1}{\gamma_{3}} \widetilde{c}^{2}+\frac{1}{\gamma_{4}} \widetilde{d}^{2}+\frac{1}{\gamma_{5}} \widetilde{f}^{2}+\frac{1}{\gamma_{6}} \widetilde{g}^{2}\right)
\end{aligned}
$$

where $\gamma_{1}, \gamma_{2}, \gamma_{3}, \gamma_{4}, \gamma_{5}$, and $\gamma_{6}$ are positive constants

The first time derivative of (50) is

$$
\begin{aligned}
\dot{V}= & e_{1} \dot{e}_{1}+e_{2} \dot{e}_{2}+e_{3} \dot{e}_{3}+\frac{1}{\gamma_{1}} \tilde{a} \dot{\tilde{a}}+\frac{1}{\gamma_{2}} \tilde{b} \dot{\tilde{b}}+\frac{1}{\gamma_{3}} \tilde{c} \dot{\tilde{c}} \\
& +\frac{1}{\gamma_{4}} \tilde{d} \dot{\tilde{d}}+\frac{1}{\gamma_{5}} \tilde{f} \dot{\tilde{f}}+\frac{1}{\gamma_{6}} \tilde{g} \dot{\tilde{g}}
\end{aligned}
$$

Or, equivalently according to (8),

$$
\begin{aligned}
\dot{V}= & e_{1} \dot{e}_{1}+e_{2} \dot{e}_{2}+e_{3} \dot{e}_{3}+\frac{1}{\gamma_{1}} \tilde{a} \dot{\hat{a}}+\frac{1}{\gamma_{2}} \tilde{b} \dot{\hat{b}}+\frac{1}{\gamma_{3}} \tilde{c} \dot{\hat{c}} \\
& +\frac{1}{\gamma_{4}} \tilde{d} \dot{\hat{d}}+\frac{1}{\gamma_{5}} \tilde{f} \dot{\hat{f}}+\frac{1}{\gamma_{6}} \tilde{g} \dot{\hat{g}}
\end{aligned}
$$




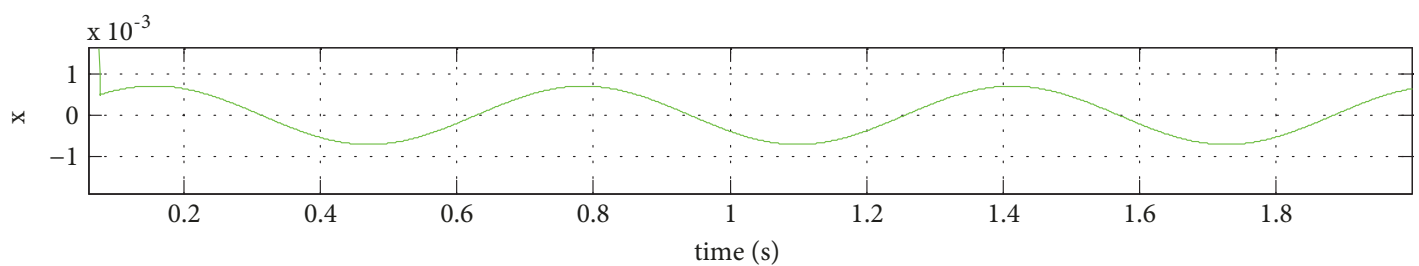

(a)

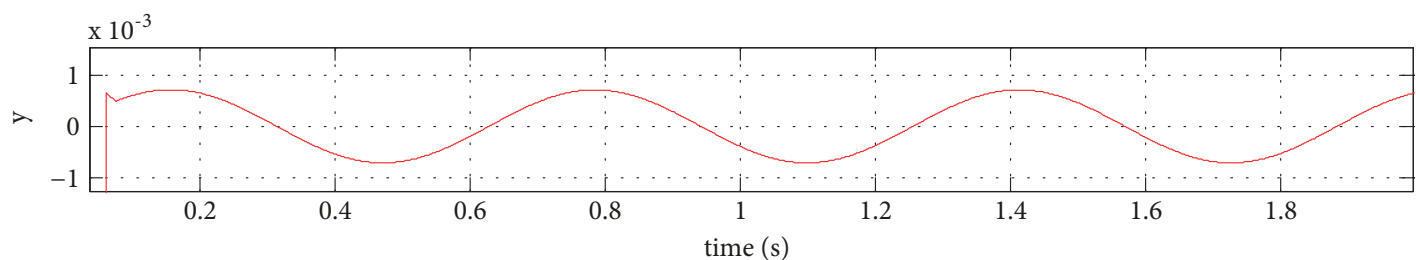

(b)

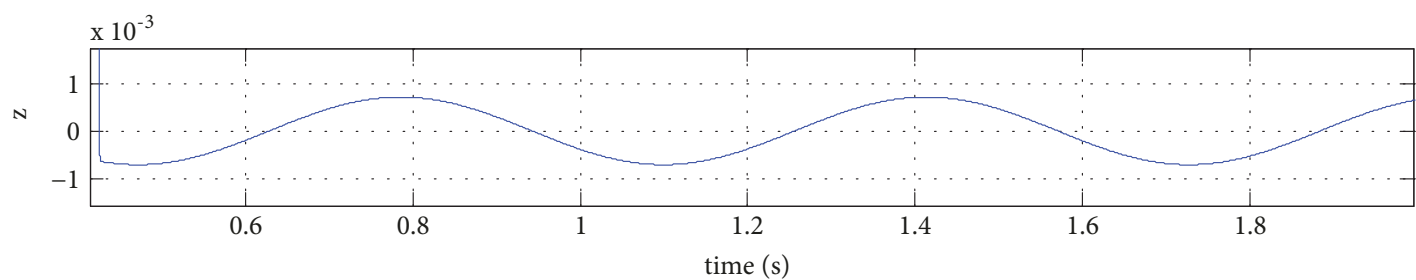

(c)

Figure 8: Zoom of Figure 7 for (a) state $x$, (b) state $y$, and (c) state $z$.

By substituting (49) into (52), and after accomplishing the corresponding operations and grouping like terms, the following is obtained:

$$
\begin{aligned}
\dot{V}= & \tilde{a}\left(e_{1}^{2}+\frac{1}{\gamma_{1}} \dot{\hat{a}}\right)+\widetilde{b}\left(-e_{1}\left(y_{s} z_{s}-y_{m} z_{m}\right)+\frac{1}{\gamma_{2}} \dot{\hat{b}}\right) \\
& +\widetilde{c}\left(e_{2}\left(y_{s}^{3}-y_{m}^{3}\right)+\frac{1}{\gamma_{3}} \dot{\hat{c}}\right) \\
& +\widetilde{d}\left(-e_{2}\left(x_{s} z_{s}-x_{m} z_{m}\right)+\frac{1}{\gamma_{4}} \dot{\hat{d}}\right) \\
& +\widetilde{f}\left(-e_{3}^{2}+\frac{1}{\gamma_{5}} \dot{\hat{f}}\right) \\
& +\widetilde{g}\left(e_{3}\left(x_{s} y_{s}-x_{m} y_{m}\right)+\frac{1}{\gamma_{6}} \dot{\hat{g}}\right)-k_{1} e_{1}^{2}-k_{2} e_{2}^{2} \\
& -k_{3} e_{3}^{2}
\end{aligned}
$$

Thus, the following learning law can be suggested:

$$
\begin{aligned}
& \dot{\hat{a}}=-\gamma_{1} e_{1}^{2} \\
& \dot{\vec{b}}=\gamma_{2} e_{1}\left(y_{s} z_{s}-y_{m} z_{m}\right) \\
& \dot{\hat{c}}=-\gamma_{3} e_{2}\left(y_{s}^{3}-y_{m}^{3}\right) \\
& \dot{\hat{d}}=\gamma_{4} e_{2}\left(x_{s} z_{s}-x_{m} z_{m}\right)
\end{aligned}
$$

$$
\begin{aligned}
& \dot{\hat{f}}=\gamma_{5} e_{3}^{2} \\
& \dot{\hat{g}}=-\gamma_{6} e_{3}\left(x_{s} y_{s}-x_{m} y_{m}\right)
\end{aligned}
$$

By substituting (54) into (53), we get

$$
\dot{V}=-k_{1} e_{1}^{2}-k_{2} e_{2}^{2}-k_{3} e_{3}^{2} \leq 0
$$

It is easy to see that the first time derivative of (50) is negative semidefinite, and then the stability properties for dynamics (47) cannot be concluded directly. From (55), it can be obtained that $V \leq V(0)$. This means that $e_{1}, e_{2}, e_{3}, \widehat{a}, \widehat{b}$, $\widehat{c}, \widehat{d}, \widehat{f}$, and $\widehat{g}$ are bounded. Now, as it is well known, the states of a chaotic autonomous system are bounded, that is, $x_{m}, y_{m}, z_{m} \in L_{\infty}$. As $e_{1}, e_{2}, e_{3} \in L_{\infty}$, and from (46), it is clear that $x_{s}, y_{s}, z_{s}$ are also bounded. Since the control law (48) is formed by bounded terms, its boundedness can be guaranteed. In a similar way, the boundedness of $\dot{e}_{1}, \dot{e}_{2}, \dot{e}_{3}$ can also be proven. From (55) and (17), the following is true:

$$
\int_{0}^{\infty}\left(e_{1}^{2}+e_{2}^{2}+e_{3}^{2}\right) d \tau \leq \frac{V(0)}{k}
$$

This means that $e_{1}, e_{2}, e_{3} \in L_{2}$. As $e_{1}, e_{2}, e_{3} \in L_{2} \cap L_{\infty}$ and $\dot{e}_{1}, \dot{e}_{2}, \dot{e}_{3} \in L_{\infty}$, from Barbalat's lemma the asymptotic convergence to zero of $e_{1}, e_{2}, e_{3}$ can be concluded. Thus, the following result has been proven:

Lemma 5. If the control law (48) with the learning law (54) is applied to Zhang slave system (4), then 


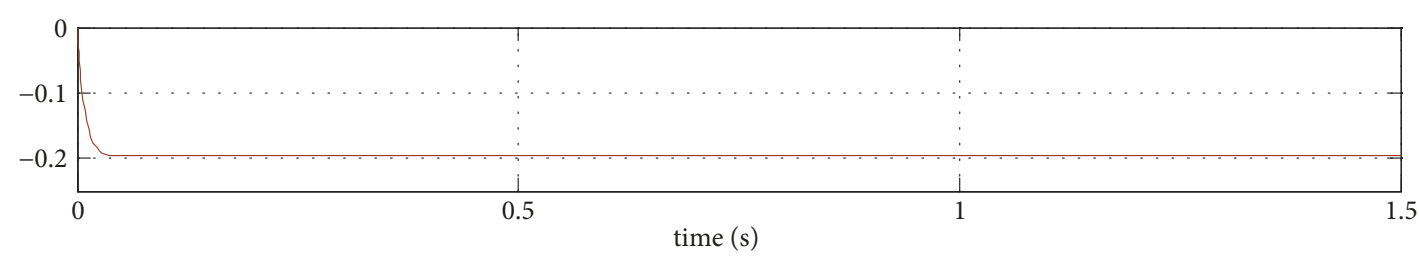

(a)

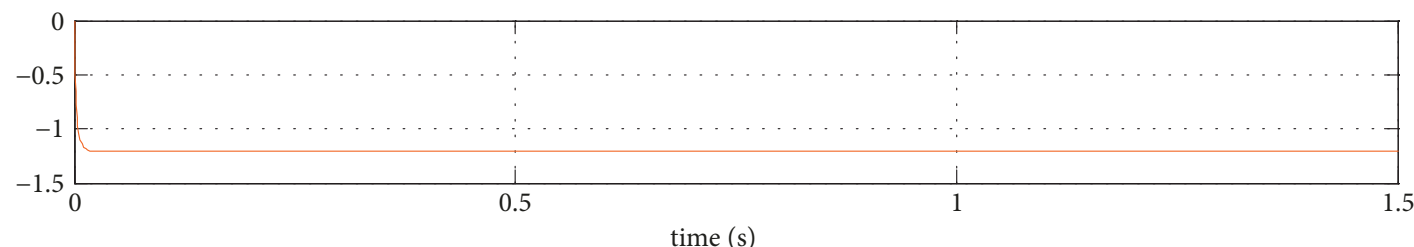

(b)

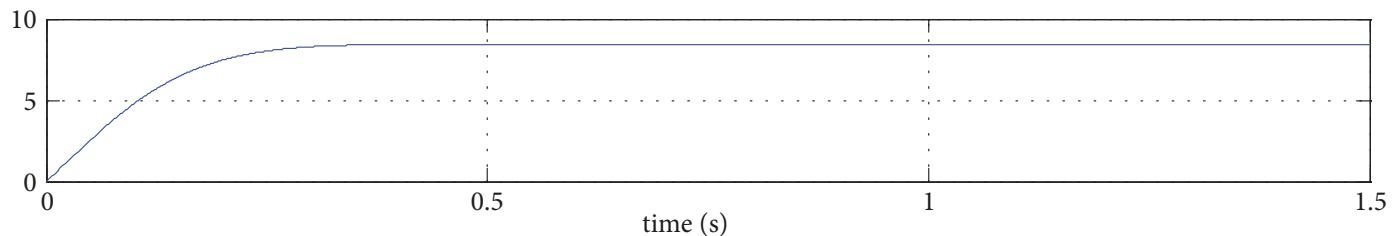

(c)

FIGURE 9: Estimations of the unknown parameters using the learning law (35): (a) $\widehat{a}$, (b) $\widehat{c}$, and (c) $\widehat{f}$.

(a) the states of slave system, the synchronization error, the estimations of the parameters, and the control signal are bounded, that is,

$x_{s}, y_{s}, z_{s}, e_{1}, e_{2}, e_{3}, \widehat{a}, \widehat{b}, \widehat{c}, \widehat{d}, \widehat{f}, \widehat{g}, u_{1}, u_{2}, u_{3} \in L_{\infty}$

(b) $e_{1}, e_{2}$, and $e_{3}$ converge asymptotically and globally to zero and consequently the synchronization between slave system (4) and master system (3) is obtained.

Corollary 2 is still valid in this new context. For the purpose of numerical simulation, consider that both slave system (4) and master system (3) have the parameters $a=2, b=10, c=$ $6.1, d=3, f=3$, and $g=1$ as depicted in Section 2. These values will be unknown to the controller. The initial condition for master system is $x_{m}(0)=0.5, y_{m}(0)=0$, and $z_{m}(0)=0.5$, whereas the initial condition for slave system is $x_{s}(0)=5$, $y_{s}(0)=-5, z_{s}(0)=7$. The gains of the controller (48) are selected as $k_{1}=k_{2}=k_{3}=1$ whereas the gains of the learning law (54) are selected as $\gamma_{1}=\gamma_{2}=\gamma_{3}=\gamma_{4}=\gamma_{5}=\gamma_{6}=1$. Finally, for simplicity, the initial condition for the learning law (54) is set up as $\widehat{a}(0)=\widehat{b}(0)=\widehat{c}(0)=\widehat{d}(0)=\widehat{f}(0)=\widehat{g}(0)=$ 0 .

The results are shown in Figure 10. In this figure, it can be appreciated as the states of the slave systems converge to the corresponding states of the master system. Clearly, the synchronization is attained in less than $1 \mathrm{~s}$. Once again, the estimations of the parameters converge to constant values. However, for briefness, this result is not presented.

4.2. Nonideal Case. In presence of unmodeled dynamics and/or disturbance, the dynamics of slave system can be described as

$$
\begin{aligned}
& \dot{x}_{s}=-a x_{s}+b y_{s} z_{s}+u_{1}+\omega_{1} \\
& \dot{y}_{s}=-c y_{s}^{3}+d x_{s} z_{s}+u_{2}+\omega_{2} \\
& \dot{z}_{s}=f z_{s}-g x_{s} y_{s}+u_{3}+\omega_{3}
\end{aligned}
$$

It is considered that Assumption 3 is still valid for $\omega_{1}$, $\omega_{2}$, and $\omega_{3}$. On these new conditions, the dynamics of synchronization error is given by

$$
\begin{aligned}
& \dot{e}_{1}=-a e_{1}+b\left(y_{s} z_{s}-y_{m} z_{m}\right)+u_{1}+\omega_{1} \\
& \dot{e}_{2}=-c\left(y_{s}^{3}-y_{m}^{3}\right)+d\left(x_{s} z_{s}-x_{m} z_{m}\right)+u_{2}+\omega_{2} \\
& \dot{e}_{3}=f e_{3}-g\left(x_{s} y_{s}-x_{m} y_{m}\right)+u_{3}+\omega_{3}
\end{aligned}
$$

If the control law (48) is used into the slave system (58), the drift of the estimations appears due to the unmodeled dynamics and/or disturbance. To avoid this problem, by generalizing the procedure used for stabilization in the presence of unmodeled dynamics/disturbances in Section 3.2, the control law (48) can be modified as follows:

$$
\begin{aligned}
u_{1}= & \widehat{a} e_{1}-\widehat{b}\left(y_{s} z_{s}-y_{m} z_{m}\right)-k_{1} e_{1}-k_{1}^{*} \operatorname{sat}\left(\frac{e_{1}}{\varepsilon_{1}}\right) \\
u_{2}= & \widehat{c}\left(y_{s}^{3}-y_{m}^{3}\right)-\widehat{d}\left(x_{s} z_{s}-x_{m} z_{m}\right)-k_{2} e_{2} \\
& -k_{2}^{*} \operatorname{sat}\left(\frac{e_{2}}{\varepsilon_{2}}\right) \\
u_{3}= & -\widehat{f} e_{3}+\widehat{g}\left(x_{s} y_{s}-x_{m} y_{m}\right)-k_{3} e_{3}-k_{3}^{*} \operatorname{sat}\left(\frac{e_{3}}{\varepsilon_{3}}\right)
\end{aligned}
$$



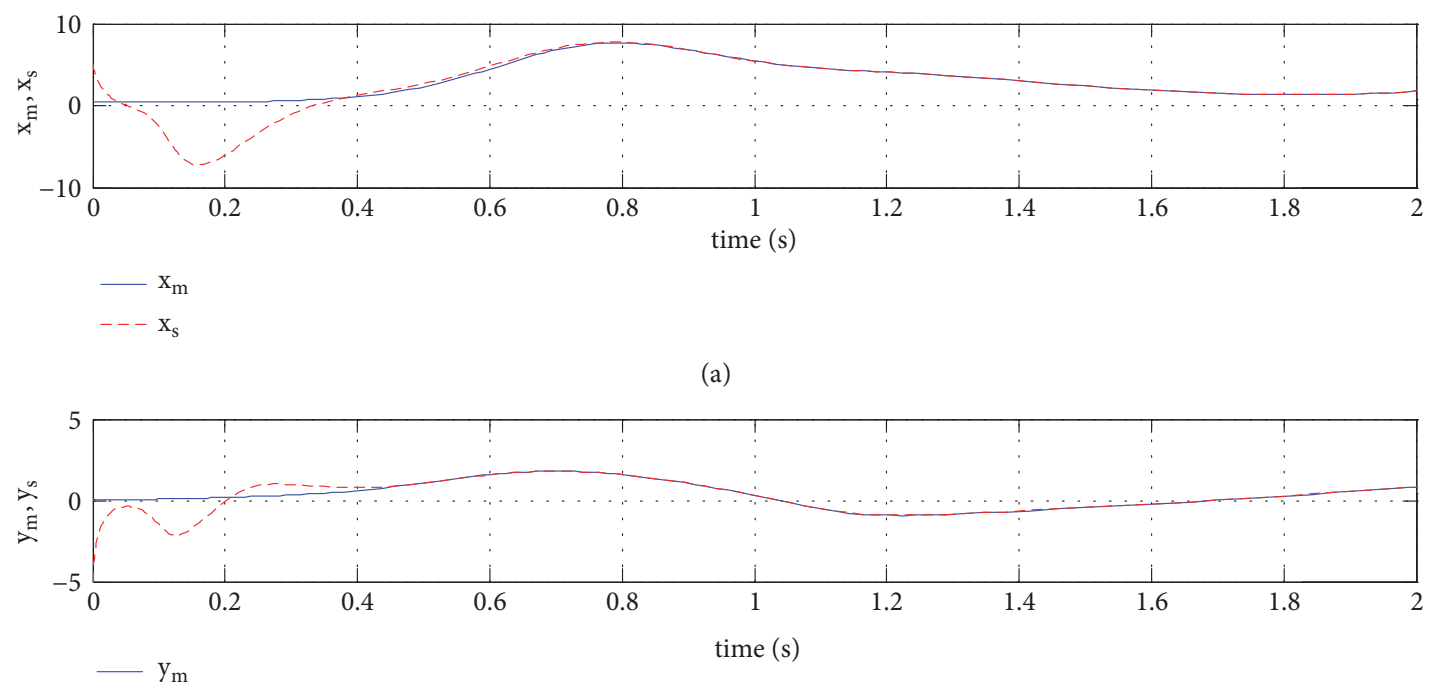

$---y_{s}$

(b)

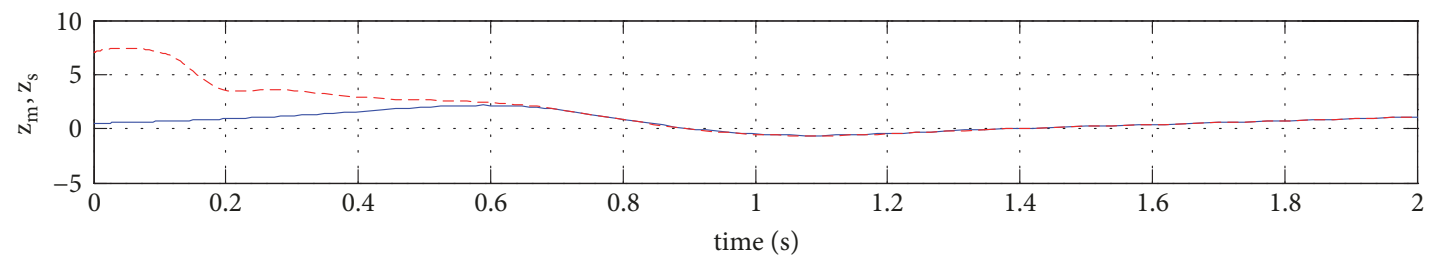

$-\mathrm{z}_{\mathrm{m}}$

(c)

FIGURE 10: Adaptive synchronization process between slave system (4) and master system (3): (a) first state, (b) second state, and (c) third state.

where $\widehat{a}, \widehat{b}, \widehat{c}, \widehat{d}, \widehat{f}$, and $\widehat{g}$ are estimations of the unknown constant parameters $a, b, c, d, f$, and $g$, respectively. $k_{1}, k_{2}$, $k_{3}, k_{1}^{*}, k_{2}^{*}, k_{3}^{*}$ are positive constants, $\varepsilon_{1}, \varepsilon_{2}$, and $\varepsilon_{3}$ are small positive constants, which can be selected by the designer, and sat $(\cdot)$ represents a saturation function as depicted in equation (27). By substituting (60) into (59), the closed-loop synchronization error dynamics is obtained as

$$
\begin{aligned}
\dot{e}_{1}= & \tilde{a} e_{1}-\widetilde{b}\left(y_{s} z_{s}-y_{m} z_{m}\right)-k_{1} e_{1}-k_{1}^{*} \operatorname{sat}\left(\frac{e_{1}}{\varepsilon_{1}}\right)+\omega_{1} \\
\dot{e}_{2}= & \widetilde{c}\left(y_{s}^{3}-y_{m}^{3}\right)-\widetilde{d}\left(x_{s} z_{s}-x_{m} z_{m}\right)-k_{2} e_{2} \\
& -k_{2}^{*} \operatorname{sat}\left(\frac{e_{2}}{\varepsilon_{2}}\right)+\omega_{2} \\
\dot{e}_{3}= & -\widetilde{f} e_{3}+\widetilde{g}\left(x_{s} y_{s}-x_{m} y_{m}\right)-k_{3} e_{3}-k_{3}^{*} \operatorname{sat}\left(\frac{e_{3}}{\varepsilon_{3}}\right) \\
& +\omega_{3}
\end{aligned}
$$

where $\widetilde{a}, \widetilde{b}, \widetilde{c}, \widetilde{d}, \widetilde{f}$, and $\widetilde{g}$ are defined as in (7). To analyze the stability of the dynamics (61), the following Lyapunov function candidate is considered:

$$
\begin{gathered}
V\left(e_{\varepsilon_{1}}, e_{\varepsilon_{2}}, e_{\varepsilon_{3}}, \widetilde{a}, \widetilde{b}, \widetilde{c}, \widetilde{d}, \widetilde{f}, \widetilde{g}\right):=\frac{1}{2}\left(e_{\varepsilon_{1}}^{2}+e_{\varepsilon_{2}}^{2}+e_{\varepsilon_{3}}^{2}\right. \\
\left.+\frac{1}{\gamma_{1}} \widetilde{a}^{2}+\frac{1}{\gamma_{2}} \widetilde{b}^{2}+\frac{1}{\gamma_{3}} \widetilde{c}^{2}+\frac{1}{\gamma_{4}} \widetilde{d}^{2}+\frac{1}{\gamma_{5}} \widetilde{f}^{2}+\frac{1}{\gamma_{6}} \widetilde{g}^{2}\right)
\end{gathered}
$$

where $\gamma_{1}, \gamma_{2}, \gamma_{3}, \gamma_{4}, \gamma_{5}$, and $\gamma_{6}$ are positive constants and

$$
\begin{aligned}
& e_{\varepsilon_{1}}:=e_{1}-\varepsilon_{1} \operatorname{sat}\left(\frac{e_{1}}{\varepsilon_{1}}\right) \\
& e_{\varepsilon_{2}}:=e_{2}-\varepsilon_{2} \operatorname{sat}\left(\frac{e_{2}}{\varepsilon_{2}}\right) \\
& e_{\varepsilon_{3}}:=e_{3}-\varepsilon_{3} \operatorname{sat}\left(\frac{e_{3}}{\varepsilon_{3}}\right)
\end{aligned}
$$

Taking into account that

$$
\begin{aligned}
& e_{\varepsilon_{1}} \dot{e}_{\varepsilon_{1}}=e_{\varepsilon_{1}} \dot{e}_{1} \\
& e_{\varepsilon_{2}} \dot{e}_{\varepsilon_{2}}=e_{\varepsilon_{2}} \dot{e}_{2} \\
& e_{\varepsilon_{3}} \dot{e}_{\varepsilon_{3}}=e_{\varepsilon_{3}} \dot{e}_{3}
\end{aligned}
$$


and according to (8), the first time derivative of (62) can be calculated as

$$
\begin{aligned}
\dot{V}= & e_{\varepsilon_{1}} \dot{e}_{1}+e_{\varepsilon_{2}} \dot{e}_{2}+e_{\varepsilon_{3}} \dot{e}_{3}+\frac{1}{\gamma_{1}} \tilde{a} \dot{\hat{a}}+\frac{1}{\gamma_{2}} \tilde{b} \dot{\hat{b}}+\frac{1}{\gamma_{3}} \tilde{c} \dot{\hat{c}} \\
& +\frac{1}{\gamma_{4}} \tilde{d} \dot{\hat{d}}+\frac{1}{\gamma_{5}} \tilde{f} \dot{\hat{f}}+\frac{1}{\gamma_{6}} \tilde{g} \dot{\hat{g}}
\end{aligned}
$$

By substituting (61) into (65), and after some operations, the following is true:

$$
\begin{aligned}
\dot{V}= & \tilde{a}\left(e_{\varepsilon_{1}} e_{1}+\frac{1}{\gamma_{1}} \dot{\hat{a}}\right) \\
& +\widetilde{b}\left(-\left(y_{s} z_{s}-y_{m} z_{m}\right) e_{\varepsilon_{1}}+\frac{1}{\gamma_{2}} \dot{\hat{b}}\right)-k_{1} e_{\varepsilon_{1}} e_{1} \\
& -e_{\varepsilon_{1}} k_{1}^{*} \operatorname{sat}\left(\frac{e_{1}}{\varepsilon_{1}}\right)+e_{\varepsilon_{1}} \omega_{1} \\
& +\widetilde{c}\left(e_{\varepsilon_{2}}\left(y_{s}^{3}-y_{m}^{3}\right)+\frac{1}{\gamma_{3}} \dot{\hat{c}}\right) \\
& +\tilde{d}\left(-e_{\varepsilon_{2}}\left(x_{s} z_{s}-x_{m} z_{m}\right)+\frac{1}{\gamma_{4}} \dot{\hat{d}}\right)-k_{2} e_{\varepsilon_{2}} e_{2} \\
& -e_{\varepsilon_{2}} k_{2}^{*} \operatorname{sat}\left(\frac{e_{2}}{\varepsilon_{2}}\right)+e_{\varepsilon_{2}} \omega_{2}+\tilde{f}\left(-e_{\varepsilon_{3}} e_{3}+\frac{1}{\gamma_{5}} \dot{\hat{f}}\right) \\
& +\tilde{g}\left(e_{\varepsilon_{3}}\left(x_{s} y_{s}-x_{m} y_{m}\right)+\frac{1}{\gamma_{6}} \dot{\hat{g}}\right)-e_{\varepsilon_{3}} k_{3} e_{3} \\
& -k_{3}^{*} e_{\varepsilon_{3}} \operatorname{sat}\left(\frac{e_{3}}{\varepsilon_{3}}\right)+e_{\varepsilon_{3}} \omega_{3}
\end{aligned}
$$

Because the objective is to get $\dot{V} \leq 0$, if the learning law is proposed,

$$
\begin{aligned}
& \dot{\hat{a}}=-\gamma_{1} e_{\varepsilon_{1}} e_{1} \\
& \dot{\hat{b}}=\gamma_{2}\left(y_{s} z_{s}-y_{m} z_{m}\right) e_{\varepsilon_{1}} \\
& \dot{\hat{c}}=-\gamma_{3} e_{\varepsilon_{2}}\left(y_{s}^{3}-y_{m}^{3}\right) \\
& \dot{\hat{d}}=\gamma_{4} e_{\varepsilon_{2}}\left(x_{s} z_{s}-x_{m} z_{m}\right) \\
& \dot{\hat{f}}=\gamma_{5} e_{\varepsilon_{3}} e_{3} \\
& \dot{\hat{g}}=-\gamma_{6} e_{\varepsilon_{3}}\left(x_{s} y_{s}-x_{m} y_{m}\right)
\end{aligned}
$$

then the following can be obtained:

$$
\begin{aligned}
\dot{V}= & -k_{1} e_{\varepsilon_{1}} e_{1}-k_{1}^{*} e_{\varepsilon_{1}} \operatorname{sat}\left(\frac{e_{1}}{\varepsilon_{1}}\right)+e_{\varepsilon_{1}} \omega_{1}-k_{2} e_{\varepsilon_{2}} e_{2} \\
& -k_{2}^{*} e_{\varepsilon_{2}} \operatorname{sat}\left(\frac{e_{2}}{\varepsilon_{2}}\right)+e_{\varepsilon_{2}} \omega_{2}-k_{3} e_{\varepsilon_{3}} e_{3} \\
& -k_{3}^{*} e_{\varepsilon_{3}} \operatorname{sat}\left(\frac{e_{3}}{\varepsilon_{3}}\right)+e_{\varepsilon_{3}} \omega_{3}
\end{aligned}
$$

Now, from (63), it is true that

$$
\begin{aligned}
& e_{1}=e_{\varepsilon_{1}}+\varepsilon_{1} \operatorname{sat}\left(\frac{e_{1}}{\varepsilon_{1}}\right) \\
& e_{2}=e_{\varepsilon_{2}}+\varepsilon_{2} \operatorname{sat}\left(\frac{e_{2}}{\varepsilon_{2}}\right) \\
& e_{3}=e_{\varepsilon_{3}}+\varepsilon_{3} \operatorname{sat}\left(\frac{e_{3}}{\varepsilon_{3}}\right)
\end{aligned}
$$

On the other hand, from Assumption 3,

$$
\begin{aligned}
& e_{\varepsilon_{1}} \omega_{1} \leq\left|e_{\varepsilon_{1}} \omega_{1}\right|=\left|e_{\varepsilon_{1}}\right|\left|\omega_{1}\right| \leq\left|e_{\varepsilon_{1}}\right| \bar{\omega}_{1} \\
& e_{\varepsilon_{2}} \omega_{2} \leq\left|e_{\varepsilon_{2}} \omega_{2}\right|=\left|e_{\varepsilon_{2}}\right|\left|\omega_{2}\right| \leq\left|e_{\varepsilon_{2}}\right| \bar{\omega}_{2} \\
& e_{\varepsilon_{3}} \omega_{3} \leq\left|e_{\varepsilon_{3}} \omega_{3}\right|=\left|e_{\varepsilon_{3}}\right|\left|\omega_{3}\right| \leq\left|e_{\varepsilon_{3}}\right| \bar{\omega}_{3}
\end{aligned}
$$

Besides, it can be proven that

$$
\begin{aligned}
& e_{\varepsilon_{1}} \text { sat }\left(\frac{e_{1}}{\varepsilon_{1}}\right)=\left|e_{\varepsilon_{1}}\right| \\
& e_{\varepsilon_{2}} \text { sat }\left(\frac{e_{2}}{\varepsilon_{2}}\right)=\left|e_{\varepsilon_{2}}\right| \\
& e_{\varepsilon_{3}} \text { sat }\left(\frac{e_{3}}{\varepsilon_{3}}\right)=\left|e_{\varepsilon_{3}}\right|
\end{aligned}
$$

By taking into account (69), (70), and (71), and after some manipulations, (68) becomes

$$
\begin{aligned}
\dot{V} \leq & -k_{1} e_{\varepsilon_{1}}^{2}-\left(k_{1} \varepsilon_{1}+k_{1}^{*}-\bar{\omega}_{1}\right)\left|e_{\varepsilon_{1}}\right|-k_{2} e_{\varepsilon_{2}}^{2} \\
& -\left(k_{2} \varepsilon_{2}+k_{2}^{*}-\bar{\omega}_{2}\right)\left|e_{\varepsilon_{2}}\right|-k_{3} e_{\varepsilon_{3}}^{2} \\
& -\left(k_{3} \varepsilon_{3}+k_{3}^{*}-\bar{\omega}_{3}\right)\left|e_{\varepsilon_{3}}\right|
\end{aligned}
$$

If the following inequality is satisfied

$$
\begin{aligned}
& k_{1} \varepsilon_{1}+k_{1}^{*}>\bar{\omega}_{1} \\
& k_{2} \varepsilon_{2}+k_{2}^{*}>\bar{\omega}_{2} \\
& k_{3} \varepsilon_{3}+k_{3}^{*}>\bar{\omega}_{3}
\end{aligned}
$$

then $\dot{V}$ can be bounded by

$$
\dot{V} \leq-k_{1} e_{\varepsilon_{1}}^{2}-k_{2} e_{\varepsilon_{2}}^{2}-k_{3} e_{\varepsilon_{3}}^{2}
$$

According to (74), $\dot{V} \leq 0$. Next, $V \leq V(0)$. This means that, due to the structure of $V$ given in (62), $e_{\varepsilon_{1}}, e_{\varepsilon_{2}}, e_{\varepsilon_{3}}, \tilde{a}, \tilde{b}, \widetilde{c}, \widetilde{d}, \widetilde{f}, \widetilde{g} \in L_{\infty}$. From (69) and (7), it can be concluded that $e_{1}, e_{2}, e_{3}, \widehat{a}, \widehat{b}, \widehat{c}, \widehat{d}, \widehat{f}, \widehat{g} \in L_{\infty}$. As $x_{m}, y_{m}, z_{m} \in$ $L_{\infty}$ and according to the definition of synchronization error (46), $x_{s}, y_{s}, z_{s} \in L_{\infty}$. It can be seen from (60) that the control law is formed by bounded terms. Thus, $u_{1}, u_{2}, u_{3} \in L_{\infty}$. As Assumption 3 is considered as still valid, from (59), $\dot{e}_{1}, \dot{e}_{2}, \dot{e}_{3} \in$ $L_{\infty}$. Likewise, from (63), $\dot{e}_{\varepsilon_{1}}, \dot{e}_{\varepsilon_{2}}, \dot{e}_{\varepsilon_{3}} \in L_{\infty}$. From (74) 


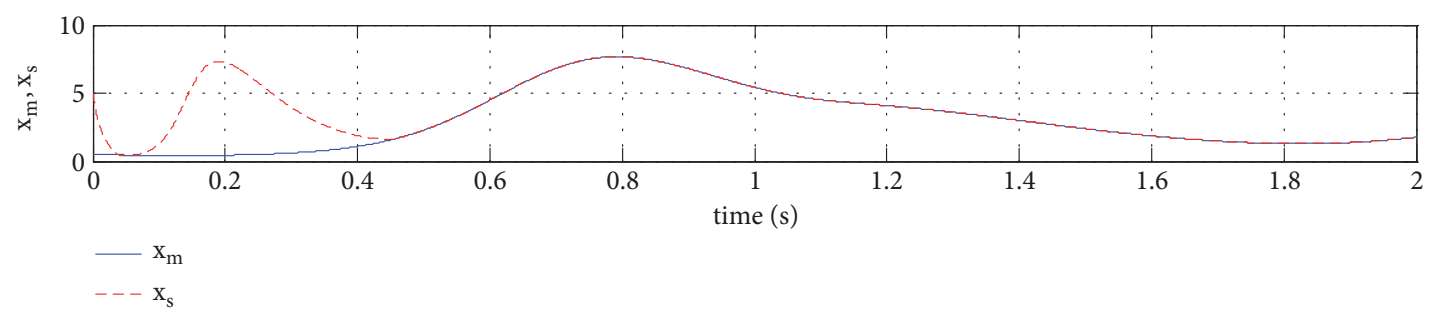

(a)

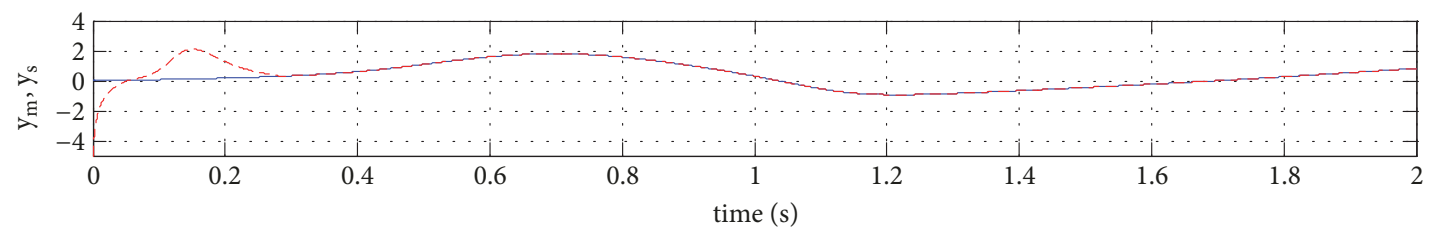

$-\mathrm{y}_{\mathrm{m}}$

$--y_{s}$

(b)

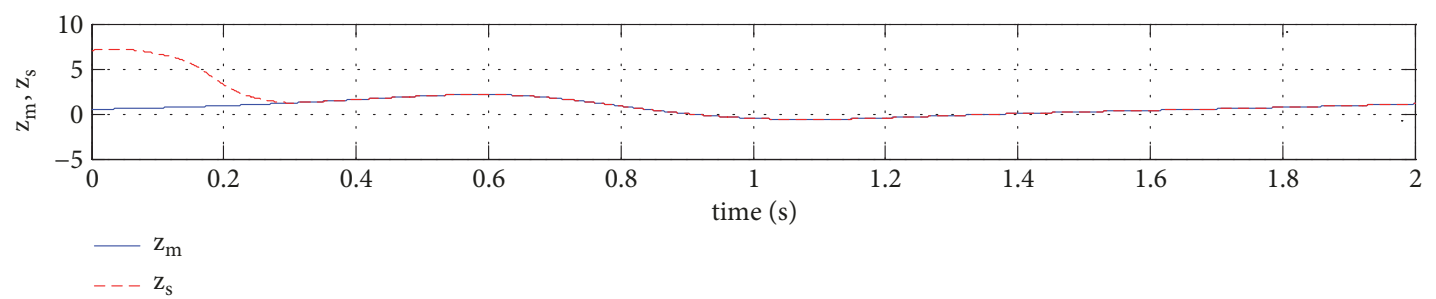

(c)

FiguRE 11: Robust adaptive synchronization process between slave system (58) and master system (3): (a) first state, (b) second state, and (c) third state.

and considering (17), $e_{\varepsilon_{1}}, e_{\varepsilon_{2}}, e_{\varepsilon_{3}}$ are quadratically integrable functions. As $e_{\varepsilon_{1}}, e_{\varepsilon_{2}}, e_{\varepsilon_{3}} \in L_{2} \cap L_{\infty}$ and $\dot{e}_{\varepsilon_{1}}, \dot{e}_{\varepsilon_{2}}, \dot{e}_{\varepsilon_{3}} \in L_{\infty}$, from Barbalat's lemma, it can be concluded that $e_{\varepsilon_{1}}, e_{\varepsilon_{2}}, e_{\varepsilon_{3}}$ converge asymptotically to zero. This means that, from (63), $e_{1}, e_{2}$, and $e_{3}$ converge asymptotically to a zone bounded by $\varepsilon_{1}, \varepsilon_{2}$, and $\varepsilon_{3}$, respectively.

Theorem 6. If Assumption (1) and inequality (73) are satisfied and the control law (60) with the learning law (67) is applied to uncertain Zhang slave system (58), then

(a) $e_{\varepsilon_{1}}, e_{\varepsilon_{2}}, e_{\varepsilon_{3}}, e_{1}, e_{2}, e_{3}, x_{s}, y_{s}, z_{s}, \widehat{a}, \widehat{b}, \widehat{c}, \widehat{d}, \widehat{f}, \widehat{g}, u_{1}, u_{2}$, $u_{3} \in L_{\infty}$

(b) $e_{1}, e_{2}$, and $e_{3}$ converge asymptotically to a region around zero bounded by $\varepsilon_{1}, \varepsilon_{2}$, and $\varepsilon_{3}$, respectively.

The effectiveness of the control law (60) can be verified using numerical simulation. The parameters for master and slave systems are as in Section 2. The initial conditions for slave and master systems are selected as in Section 4.1. The following values are used for the parameters of the control law (60) and the learning law (67), respectively, $\varepsilon_{1}=\varepsilon_{2}=\varepsilon_{3}=0.001$, $k_{1}=k_{2}=k_{3}=1, k_{1}^{*}=k_{2}^{*}=k_{3}^{*}=7, \gamma_{1}=\gamma_{2}=\gamma_{3}=\gamma_{4}=\gamma_{5}=$ $\gamma_{6}=1$, and $\widehat{a}(0)=\widehat{b}(0)=\widehat{c}(0)=\widehat{d}(0)=\widehat{f}(0)=\widehat{g}(0)=$ 0 . Besides, the unmodeled dynamics and/or disturbance is assumed as $\omega_{1}=\omega_{2}=\omega_{3}=5 \sin (10 t)$. The results are shown in Figures 11, 12, and 13. In Figure 11, it can be appreciated that the states of slave system follow the corresponding states of the master system. In Figure 12, the synchronization error signals $e_{1}, e_{2}$, and $e_{3}$ are displayed. Although the convergence to zero of the synchronization error cannot be obtained due to the presence of unmodeled dynamics and/or disturbance, now the synchronization error converges to a bounded zone around zero less than 0.001 . Besides, the convergence to this bounded zone is attained in less than $0.5 \mathrm{~s}$. This is shown in Figure 13.

\section{Conclusion}

In this paper, based on a Lyapunov-like approach, a conventional adaptive control law was designed for stabilization of the uncertain chaotic Zhang system. The performance obtained by this controller was adequate for the ideal case. However, under the presence of unmodeled dynamics and/or disturbance, this controller was not effective anymore since the parameter estimations started to grow unboundedly. This effect, known as drift, was illustrated by simulation. As the drift causes the instability of the closed-loop system, a saturation function was added to the conventional control law to overcome this problem. The learning law was also modified. The boundedness of the parameter estimations was rigorously proven. Numerical simulation showed the 


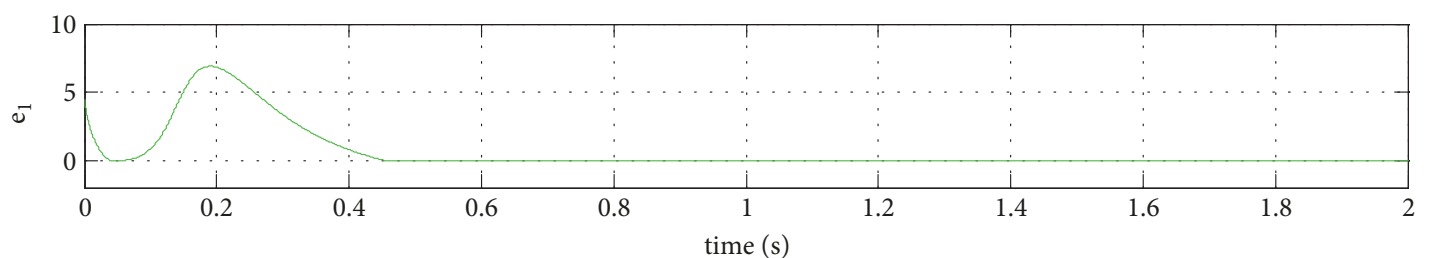

(a)

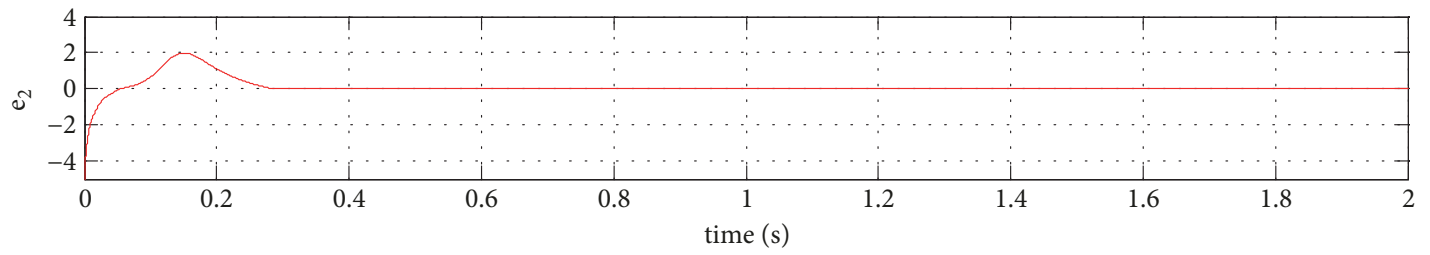

(b)

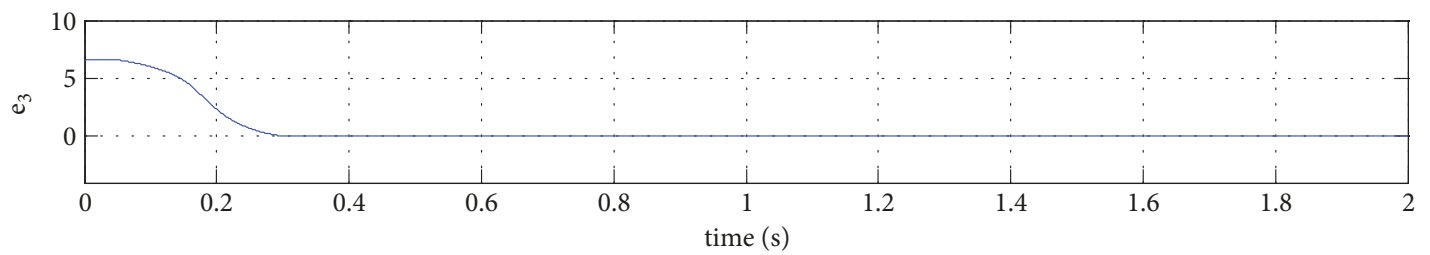

(c)

FIGURE 12: Time evolution of synchronization error signals: (a) $e_{1}$, (b) $e_{2}$, and (c) $e_{3}$.

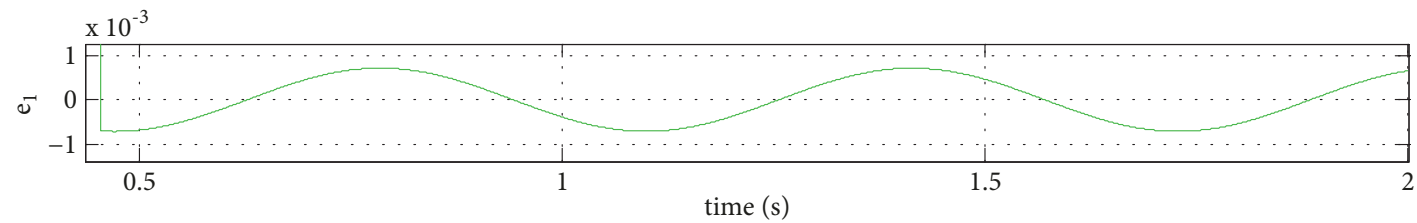

(a)

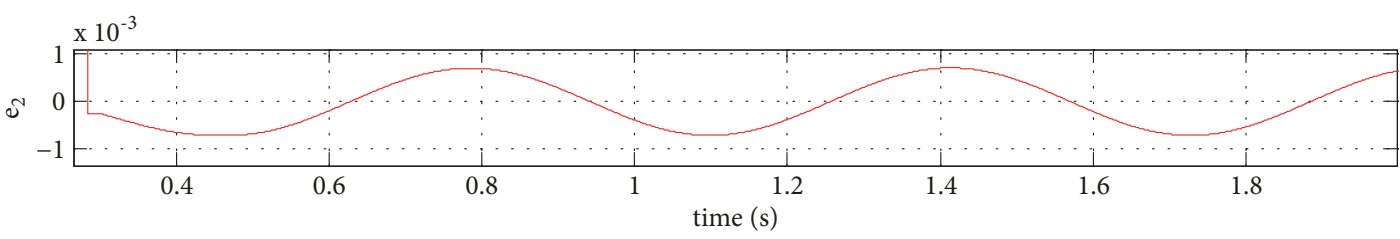

(b)

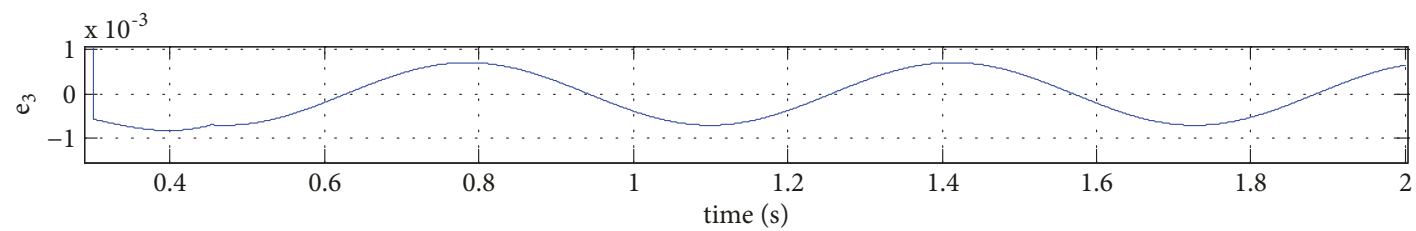

(c)

FIGURE 13: Zoom of Figure 12 for (a) $e_{1}$, (b) $e_{2}$, and (c) $e_{3}$.

satisfactory performance of this robust adaptive control law. The states of the chaotic system converged to a bounded zone (instead of zero as in the ideal case). Likewise, the synchronization between a slave system and a master system both based on Zhang system was considered. Two cases were studied: (a) ideal case and (b) case under the presence of unmodeled dynamics and/or disturbance. For the first case, a standard control law was developed. For the nonideal case, this control law had to be modified by adding a saturation function. By means of this modification, the drift was successfully avoided. However, it was not possible to obtain the asymptotic convergence to zero of the synchronization error. 
Only it was possible to guarantee the convergence of this error to a bounded zone. Numerical simulations confirmed the effectiveness of the approach. It should be mentioned that although the width of the bounded zone can be adjusted by the designer, a very small zone could cause chattering in the control law. Consequently, a compromise should be established between these conflicting goals.

\section{Data Availability}

No experimental data were used to support this study. The simulation results can be reproduced with Matlab and/or Simulink just using the mathematical model of Zhang system and proposed controllers given in Sections 3 and 4.

\section{Conflicts of Interest}

The author declares that there are no conflicts of interest regarding the publication of this article.

\section{Acknowledgments}

The author acknowledges the support of EDI-IPN, and SNIConacyt. This work was financed by SIP Instituto Politécnico Nacional [grant number 20180054].

\section{References}

[1] E. N. Lorenz, "Deterministic nonperiodic flow," Journal of the Atmospheric Sciences, vol. 20, no. 2, pp. 130-141, 1963.

[2] H. S. Nik, S. Effati, and J. Saberi-Nadjafi, "Ultimate bound sets of a hyperchaotic system and its application in chaos synchronization," Complexity, vol. 20, pp. 30-44, 2015.

[3] H. Tirandaz and S. Saeidiaminabadi, "Identical and Nonidentical Synchronization of Three Scroll Unified Chaotic System (TSUCS) with Unknown Parameter Using a Modified Function Projective Control Method," Iranian Journal of Science and Technology - Transactions of Electrical Engineering, vol. 41, no. 4, pp. 319-334, 2017.

[4] E. Tlelo-Cuautle, L. G. de la Fraga, V.-T. Pham, C. Volos, S. Jafari, and A. D. J. Quintas-Valles, "Dynamics, FPGA realization and application of a chaotic system with an infinite number of equilibrium points," Nonlinear Dynamics, vol. 89, no. 2, pp. 1129-1139, 2017.

[5] W. Gao, L. Yan, M. Saeedi, and H. Saberi Nik, "Ultimate bound estimation set and chaos synchronization for a financial risk system," Mathematics and Computers in Simulation, vol. 154, pp. 19-33, 2018.

[6] Q. Lai, A. Akgul, C. Li, G. Xu, and Ü. Çavuşoğlu, "A new chaotic system with multiple attractors: Dynamic analysis, circuit realization and S-Box design," Entropy, vol. 20, no. 1, 2018.

[7] P. Glorieux, "Control of chaos in lasers by feedback and nonfeedback methods," International Journal of Bifurcation and Chaos, vol. 8, no. 9, pp. 1749-1758, 1998.

[8] S. Yan, "Control of chaos in an external-cavity multiquantumwell laser subjected to dual-wedges and optical dual-feedback," Chinese Science Bulletin, vol. 54, no. 7, pp. 1158-1163, 2009.
[9] G. Yuan, X. Zhang, and Z. Wang, "Generation and synchronization of feedback-induced chaos in semiconductor ring lasers by injection-locking," Optik - International Journal for Light and Electron Optics, vol. 125, no. 8, pp. 1950-1953, 2014.

[10] D. Ghosh, A. Mukherjee, N. R. Das, and B. N. Biswas, "Generation \& control of chaos in a single loop optoelectronic oscillator," Optik - International Journal for Light and Electron Optics, vol. 165, pp. 275-287, 2018.

[11] J. M. Grzybowski, M. Rafikov, and J. M. Balthazar, "Synchronization of the unified chaotic system and application in secure communication," Communications in Nonlinear Science and Numerical Simulation, vol. 14, no. 6, pp. 2793-2806, 2009.

[12] A. K. Mittal, A. Dwivedi, and S. Dwivedi, "Secure communication based on chaotic switching and rapid synchronization using parameter adaptation," International Journal of Innovative Computing, Information and Control, vol. 11, no. 2, pp. 569-585, 2015.

[13] E. E. Mahmoud and S. M. Abo-Dahab, "Dynamical properties and complex anti synchronization with applications to secure communications for a novel chaotic complex nonlinear model," Chaos, Solitons \& Fractals, vol. 106, pp. 273-284, 2018.

[14] M. Zapateiro De La Hoz, L. Acho, and Y. Vidal, "An experimental realization of a chaos-based secure communication using Arduino microcontrollers," The Scientific World Journal, vol. 2015, Article ID 123080, p. 10, 2015.

[15] J. He, J. Cai, and J. Lin, "Synchronization of hyperchaotic systems with multiple unknown parameters and its application in secure communication," Optik - International Journal for Light and Electron Optics, vol. 127, no. 5, pp. 2502-2508, 2016.

[16] R. Martínez-Guerra, J. J. Montesinos García, and S. M. Delfín Prieto, "Secure communications via synchronization of Liouvillian chaotic systems," Journal of The Franklin Institute, vol. 353, no. 17, pp. 4384-4399, 2016.

[17] H. Tirandaz and A. Karmi-Mollaee, "Modified function projective feedback control for time-delay chaotic Liu system synchronization and its application to secure image transmission," Optik - International Journal for Light and Electron Optics, vol. 147, pp. 187-196, 2017.

[18] G. Xu, J. Xu, C. Xiu, F. Liu, and Y. Zang, "Secure communication based on the synchronous control of hysteretic chaotic neuron," Neurocomputing, vol. 227, pp. 108-112, 2017.

[19] Z. Sun, L. Si, Z. Shang, and J. Lei, "Finite-time synchronization of chaotic PMSM systems for secure communication and parameters identification," Optik - International Journal for Light and Electron Optics, vol. 157, pp. 43-55, 2018.

[20] D. Chang, Z. Li, M. Wang, and Y. Zeng, "A novel digital programmable multi-scroll chaotic system and its application in FPGA-based audio secure communication," AEÜ - International Journal of Electronics and Communications, vol. 88, pp. 20-29, 2018.

[21] I. Ahmad, M. Shafiq, and M. M. Al-Sawalha, "Globally exponential multi switching-combination synchronization control of chaotic systems for secure communications," Chinese Journal of Physics, vol. 56, no. 3, pp. 974-987, 2018.

[22] N. Vafamand, S. Khorshidi, and A. Khayatian, "Secure communication for non-ideal channel via robust TS fuzzy observerbased hyperchaotic synchronization," Chaos Solitons \& Fractals, vol. 112, pp. 116-124, 2018.

[23] N. Vafamand and S. Khorshidi, "Robust Polynomial ObserverBased Chaotic Synchronization for Non-ideal Channel Secure Communication: An SOS Approach," Iranian Journal of Science 
and Technology, Transactions of Electrical Engineering, vol. 42, no. 1, pp. 83-94, 2018.

[24] G. Chaparro Guevara and L. Escot Mangas, "El control de sistemas dinámicos caóticos en economía: aplicación a un modelo de hiperinflación," Revista Finanzas y Política Económica, pp. 131-145, 2015.

[25] O. I. Tacha, C. K. Volos, I. M. Kyprianidis, I. N. Stouboulos, S. Vaidyanathan, and V.-T. Pham, "Analysis, adaptive control and circuit simulation of a novel nonlinear finance system," Applied Mathematics and Computation, vol. 276, pp. 200-217, 2016.

[26] S. Vaidyanathan, C. K. Volos, O. I. Tacha, I. M. Kyprianidis, I. N. Stouboulos, and V.-T. Pham, "Analysis, control and circuit simulation of a novel 3-D finance chaotic system," in Adv. Appl. Chaotic Syst, pp. 495-512, Springer, Cham, Switzerland, 2016.

[27] H. Tirandaz, S. S. Aminabadi, and H. Tavakoli, "Chaos synchronization and parameter identification of a finance chaotic system with unknown parameters, a linear feedback controller," Alexandria Engineering Journal, 2016.

[28] A. Jajarmi, M. Hajipour, and D. Baleanu, "New aspects of the adaptive synchronization and hyperchaos suppression of a financial model," Chaos, Solitons \& Fractals, vol. 99, pp. 285296, 2017.

[29] I. M. Ginarsa, A. Soeprijanto, and M. H. Purnomo, "Controlling chaos and voltage collapse using an ANFIS-based composite controller-static var compensator in power systems," International Journal of Electrical Power \& Energy Systems, vol. 46, no. 1, pp. 79-88, 2013.

[30] M. S. Sadeghi, N. Vafamand, and M. . Khooban, "LMI-based stability analysis and robust controller design for a class of nonlinear chaotic power systems," Journal of The Franklin Institute, vol. 353, no. 13, pp. 2835-2858, 2016.

[31] H. Ma, F. Min, and Y. Wang, "Nonlinear dynamic analysis and surface sliding mode controller based on low pass filter for chaotic oscillation in power system with power disturbance," Chinese Journal of Physics, vol. 56, no. 5, pp. 2488-2499, 2018.

[32] C. Wang, H. Zhang, W. Fan, and P. Ma, "Adaptive control method for chaotic power systems based on finite-time stability theory and passivity-based control approach," Chaos, Solitons \& Fractals, vol. 112, pp. 159-167, 2018.

[33] N. Vafamand, M. H. Khooban, A. Khayatian, and F. Blabbjerg, "Design of Robust Double-Fuzzy-Summation Nonparallel Distributed Compensation Controller for Chaotic Power Systems," Journal of Dynamic Systems, Measurement, and Control, vol. 140, no. 3, p. 031004, 2018.

[34] M. Zribi, A. Oteafy, and N. Smaoui, "Controlling chaos in the permanent magnet synchronous motor," Chaos, Solitons \& Fractals, vol. 41, no. 3, pp. 1266-1276, 2009.

[35] M. Messadi and A. Mellit, "Control of chaos in an induction motor system with LMI predictive control and experimental circuit validation," Chaos, Solitons \& Fractals, vol. 97, pp. 51-58, 2017.

[36] S. Luo and R. Gao, "Chaos control of the permanent magnet synchronous motor with time-varying delay by using adaptive sliding mode control based on DSC," Journal of The Franklin Institute, vol. 355, no. 10, pp. 4147-4163, 2018.

[37] J. A. Rodríguez and J. De León, "Control de sistemas caóticos," Universidad Autónoma de Nuevo León, vol. 1, pp. 29-35, 2008.

[38] J. H. Perez, "Neural control for synchronization of a chaotic Chua-Chen system," IEEE Latin America Transactions, vol. 14, no. 8, pp. 3560-3568, 2016.
[39] C. Aguilar-Ibañez, R. Martinez-Guerra, R. Aguilar-López, and J. L. Mata-Machuca, "Synchronization and parameter estimations of an uncertain Rikitake system," Physics Letters A, vol. 374, no. 35, pp. 3625-3628, 2010.

[40] F. Motallebzadeh, M. R. Jahed Motlagh, and Z. Rahmani Cherati, "Synchronization of different-order chaotic systems: adaptive active vs. optimal control," Communications in Nonlinear Science and Numerical Simulation, vol. 17, no. 9, pp. 36433657, 2012.

[41] L. M. Pecora and T. L. Carroll, "Synchronization of chaotic systems," Chaos: An Interdisciplinary Journal of Nonlinear Science, vol. 25, no. 9, Article ID 097611, 12 pages, 2015.

[42] K.-B. Deng, R.-X. Wang, C.-L. Li, and Y.-Q. Fan, "Tracking control for a ten-ring chaotic system with an exponential nonlinear term," Optik - International Journal for Light and Electron Optics, vol. 130, pp. 576-583, 2017.

[43] S. Vaidyanathan and A. Rhif, "A novel four-leaf chaotic system, its control and synchronisation via integral sliding mode control," International Journal of Modelling, Identification and Control, vol. 28, no. 1, pp. 28-39, 2017.

[44] J. Cai and M. Ma, "Synchronization between two nonautonomous chaotic systems via intermittent control of sinusoidal state error feedback," Optik - International Journal for Light and Electron Optics, vol. 130, pp. 455-463, 2017.

[45] B. Wang and S. Zhong, "Observer-based control on a chaotic system with unknowns and uncertainties," Optik - International Journal for Light and Electron Optics, vol. 137, pp. 167-174, 2017.

[46] M. Varan and A. Akgul, "Control and synchronisation of a novel seven-dimensional hyperchaotic system with active control," Pramana-Journal of Physics, vol. 90, no. 4, 2018.

[47] Q. Ye, Z. Jiang, and T. Chen, "Adaptive Feedback Control for Synchronization of Chaotic Neural Systems with Parameter Mismatches," Complexity, vol. 2018, Article ID 5431987, 8 pages, 2018.

[48] M. M. Mardani, N. Vafamand, M. Shokrian Zeini, M. Shasadeghi, and A. Khayatian, "Sum-of-squares-based finitetime adaptive sliding mode control of uncertain polynomial systems with input nonlinearities," Asian Journal of Control, vol. 20, no. 4, pp. 1658-1662, 2018.

[49] Z. Y. Yan and P. Yu, "Linear feedback control, adaptive feedback control and their combination for chaos (lag) synchronization of LC chaotic systems," Chaos, Solitons \& Fractals, vol. 33, no. 2, pp. 419-435, 2007.

[50] X. Wu, G. Chen, and J. Cai, "Chaos synchronization of the master-slave generalized Lorenz systems via linear state error feedback control," Physica D: Nonlinear Phenomena, vol. 229, no. 1, pp. 52-80, 2007.

[51] M. Rafikov and J. M. Balthazar, "On control and synchronization in chaotic and hyperchaotic systems via linear feedback control," Communications in Nonlinear Science and Numerical Simulation, vol. 13, no. 7, pp. 1246-1255, 2008.

[52] Y. Chen, X. Wu, and Z. Gui, "Global synchronization criteria for a class of third-order non-autonomous chaotic systems via linear state error feedback control," Applied Mathematical Modelling: Simulation and Computation for Engineering and Environmental Systems, vol. 34, no. 12, pp. 4161-4170, 2010.

[53] U. E. Kocamaz, Y. Uyaroğlu, and H. Kizmaz, "Controlling hyperchaotic Rabinovich system with single state controllers: Comparison of linear feedback, sliding mode, and passive control methods," Optik - International Journal for Light and Electron Optics, vol. 130, pp. 914-921, 2017. 
[54] M. Chen and Z. Han, "Controlling and synchronizing chaotic Genesio system via nonlinear feedback control," Chaos, Solitons \& Fractals, vol. 17, no. 4, pp. 709-716, 2003.

[55] L. Huang, R. Feng, and M. Wang, "Synchronization of chaotic systems via nonlinear control," Physics Letters A, vol. 320, no. 4, pp. 271-275, 2004.

[56] H.-K. Chen, "Global chaos synchronization of new chaotic systems via nonlinear control," Chaos, Solitons \& Fractals, vol. 23, no. 4, pp. 1245-1251, 2005.

[57] Q. Zhang and J. an Lu, "Chaos synchronization of a new chaotic system via nonlinear control," Chaos Solitons and Fractals, vol. 37, pp. 175-179, 2008.

[58] J. H. Pérez-Cruz, E. A. Portilla-Flores, P. A. Niño-Suárez, and R. Rivera-Blas, "Design of a nonlinear controller and its intelligent optimization for exponential synchronization of a new chaotic system," Optik - International Journal for Light and Electron Optics, vol. 130, pp. 201-212, 2017.

[59] U. E. Vincent, "Synchronization of Rikitake chaotic attractor using active control," Physics Letters A, vol. 343, no. 1-3, pp. 133138, 2005.

[60] Y. M. Lei, W. Xu, and H. C. Zheng, "Synchronization of two chaotic nonlinear gyros using active control," Physics Letters A, vol. 343, no. 1-3, pp. 153-158, 2005.

[61] A. Uçar, K. E. Lonngren, and E.-W. Bai, "Synchronization of the unified chaotic systems via active control," Chaos, Solitons \& Fractals, vol. 27, no. 5, pp. 1292-1297, 2006.

[62] U. Vincent and J. Laoye, "Synchronization and control of directed transport in chaotic ratchets via active control," Physics Letters A, vol. 363, no. 1-2, pp. 91-95, 2007.

[63] Y. Lei, W. Xu, and W. Xie, "Synchronization of two chaotic fourdimensional systems using active control," Chaos, Solitons \& Fractals, vol. 32, no. 5, pp. 1823-1829, 2007.

[64] U. E. Vincent, "Synchronization of identical and non-identical 4-D chaotic systems using active control," Chaos, Solitons \& Fractals, vol. 37, no. 4, pp. 1065-1075, 2008.

[65] V. Sundarapandian and R. Karthikeyan, "Hybrid synchronization of hyperchaotic lorenz and hyperchaotic chen systems via active control," Journal of Engineering and Applied Sciences, vol. 7, no. 3, pp. 254-264, 2012.

[66] P. P. Singh, J. P. Singh, and B. K. Roy, "Synchronization and antisynchronization of Lu and Bhalekar-Gejji chaotic systems using nonlinear active control," Chaos, Solitons \& Fractals, vol. 69, pp. 31-39, 2014.

[67] S. Çiçek, A. Ferikoğlu, and I. Pehlivan, "A new 3D chaotic system: dynamical analysis, electronic circuit design, active control synchronization and chaotic masking communication application," Optik - International Journal for Light and Electron Optics, vol. 127, no. 8, pp. 4024-4030, 2016.

[68] P. Singh and B. Roy, "Comparative performances of synchronisation between different classes of chaotic systems using three control techniques," Annual Reviews in Control, vol. 45, pp. 152$165,2018$.

[69] J. H. Pérez-Cruz, D. Zenteno-Lara, D. Ávila-González, C. Nwachioma, J. H. Pérez-Cruz, and D. Ávila-González, "Synchronization of Multi-character Nazarimehr System using Active Control," International Journal of Scientific and Engineering Research, vol. 9, pp. 438-444, 2018.

[70] J. H. Pérez, M. Figueroa, S. A. Rodríguez, and A. López, "Synchronization of chaotic Akgul system by means of feedback linearization and pole placement," IEEE Latin America Transactions, vol. 15, no. 2, pp. 249-256, 2017.
[71] M. M. Al-Sawalha, "Adaptive synchronization and antisynchronization of a chaotic lorenz - Stenflo system with fully unknown parameters," Chinese Physics Letters, vol. 30, no. 7, 2013.

[72] A. Akgul, I. Moroz, I. Pehlivan, and S. Vaidyanathan, "A new four-scroll chaotic attractor and its engineering applications," Optik - International Journal for Light and Electron Optics, vol. 127, no. 13, pp. 5491-5499, 2016.

[73] H. Tirandaz and A. Hajipour, "Adaptive synchronization and anti-synchronization of TSUCS and Lü unified chaotic systems with unknown parameters," Optik - International Journal for Light and Electron Optics, vol. 130, pp. 543-549, 2017.

[74] X. Wang, V.-T. Pham, S. Jafari, C. Volos, J. M. Munoz-Pacheco, and E. Tlelo-Cuautle, "A New Chaotic System with Stable Equilibrium: From Theoretical Model to Circuit Implementation," IEEE Access, vol. 5, pp. 8851-8858, 2017.

[75] H. Tirandaz, "On Complete Control and Synchronization of Zhang Chaotic System with Uncertain Parameters using Adaptive Control Method," Nonlinear Engineering, vol. 7, pp. 45-50, 2017.

[76] H. Zhang, W. Huang, Z. Wang, and T. Chai, "Adaptive synchronization between two different chaotic systems with unknown parameters," Physics Letters A, vol. 350, no. 5-6, pp. 363-366, 2006.

[77] M. Yassen, "Adaptive chaos control and synchronization for uncertain new chaotic dynamical system," Physics Letters A, vol. 350, no. 1-2, pp. 36-43, 2006.

[78] X. Wu, Z.-H. Guan, Z. Wu, and T. Li, "Chaos synchronization between Chen system and Genesio system," Physics Letters A, vol. 364, no. 6, pp. 484-487, 2007.

[79] J. Huang, "Chaos synchronization between two novel different hyperchaotic systems with unknown parameters," Nonlinear Analysis. Theory, Methods \& Applications, vol. 69, no. 11, pp. 4174-4181, 2008.

[80] S. Vaidyanathan, "Adaptive control and synchronization of Rössler prototype-4 system," International Journal of Advanced Information Technology, vol. 1, 2011.

[81] M. Zhang and Q. Han, "Dynamic analysis of an autonomous chaotic system with cubic nonlinearity," Optik - International Journal for Light and Electron Optics, vol. 127, no. 10, pp. 43154319, 2016.

[82] A. S. Poznyak, E. N. Sanchez, and W. Yu, Differential Neural Networks for Robust Nonlinear Control-Identification, State Estimation, and Trajectory Tracking, WORLD SCIENTIFIC, 1st edition, 2001.

[83] X.-S. Wang, C.-Y. Su, and H. Hong, "Robust adaptive control of a class of nonlinear systems with unknown dead-zone," Automatica, vol. 40, no. 3, pp. 407-413, 2004. 


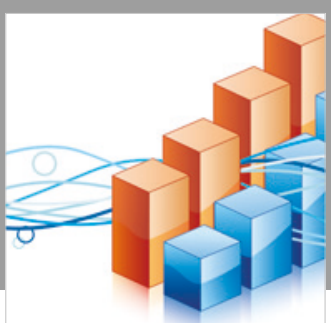

Advances in

Operations Research

\section{-n-m}
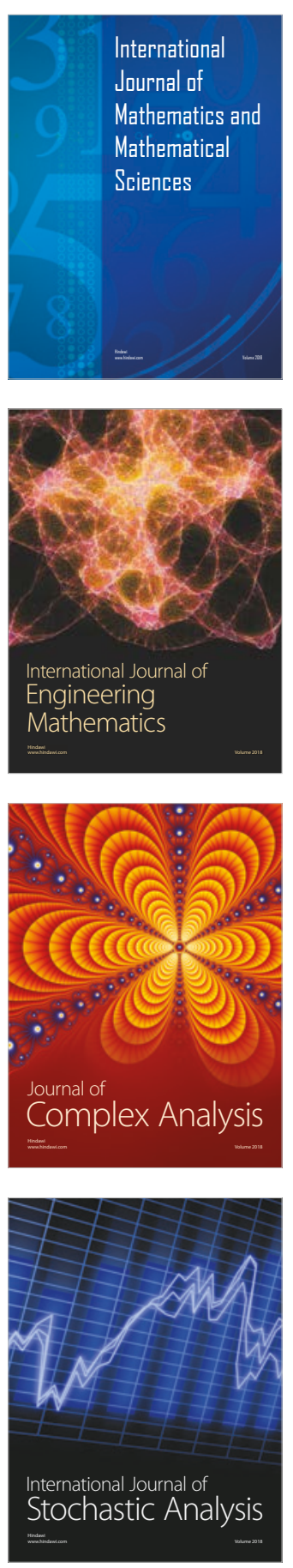
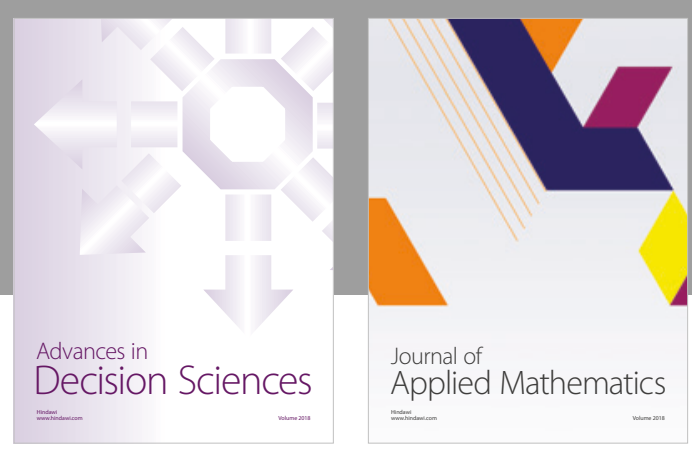

Journal of

Applied Mathematics
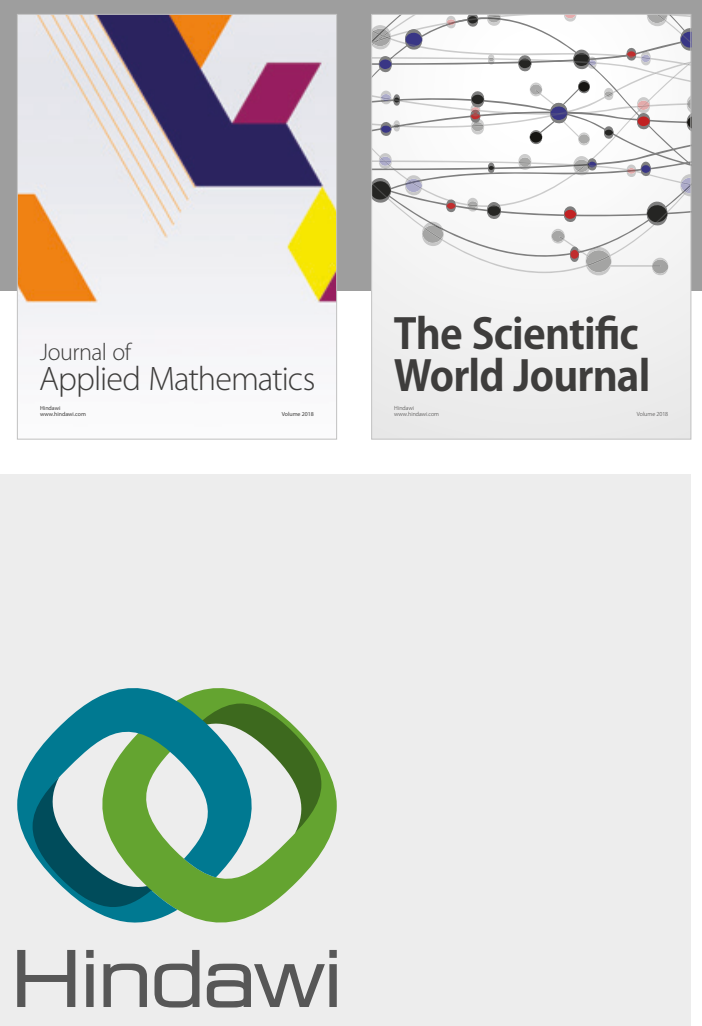

Submit your manuscripts at

www.hindawi.com

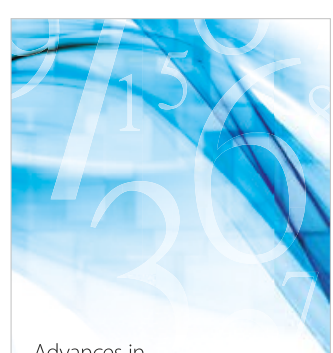

Advances in
Numerical Analysis
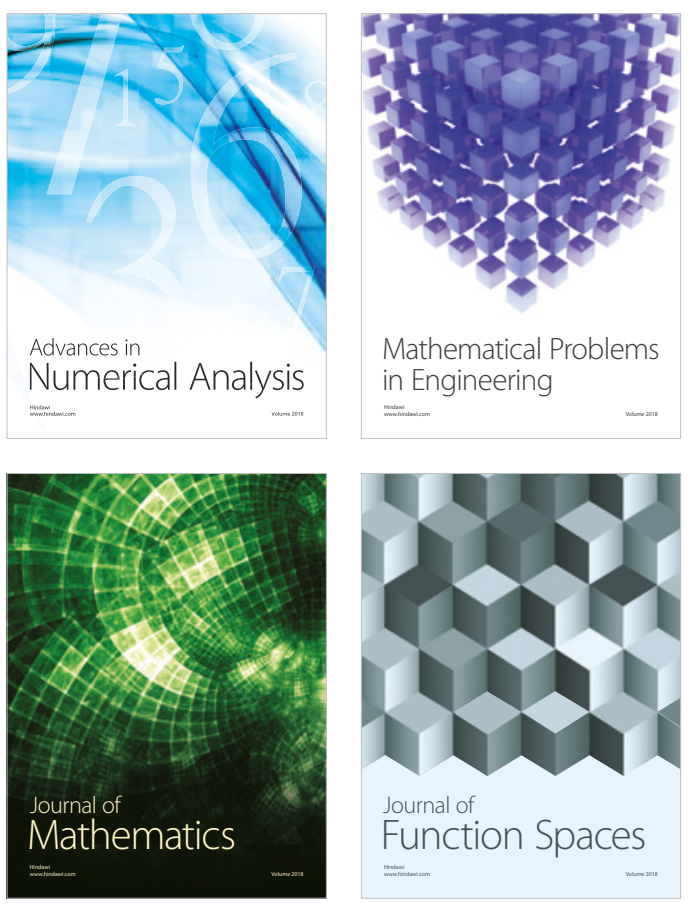

Mathematical Problems in Engineering

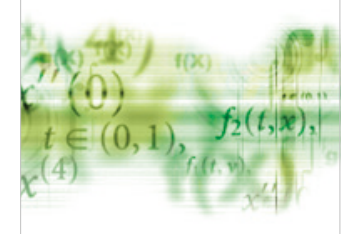

International Journal of

Differential Equations

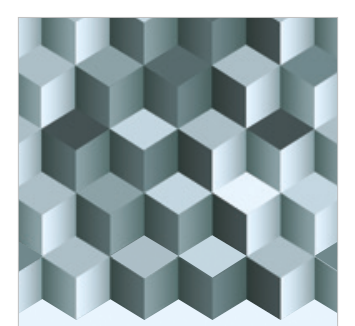

Journal of

Function Spaces
The Scientific

World Journal

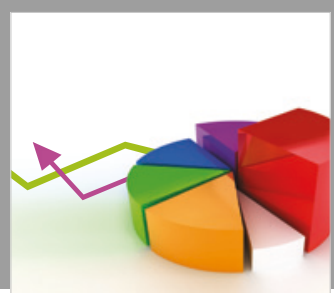

Journal of

Probability and Statistics
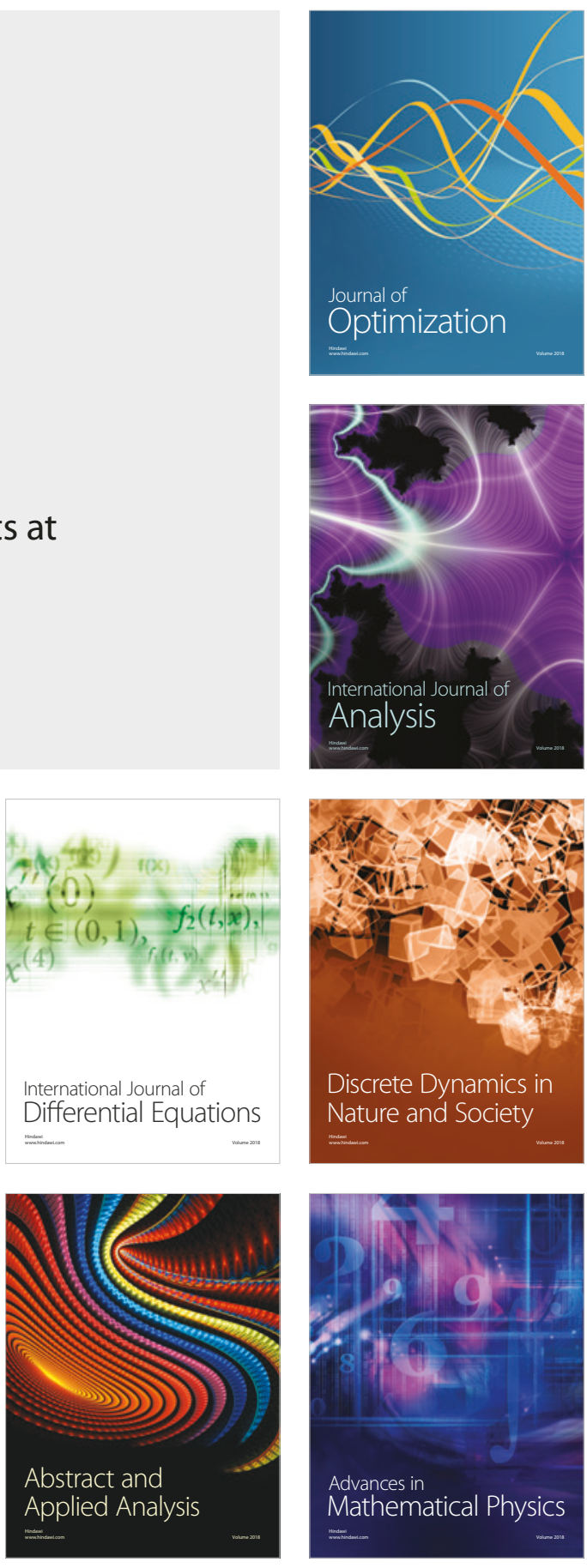